\title{
A quantitative demonstration that stellar feedback locally regulates galaxy growth
}

\author{
Javier Zaragoza-Cardiel, ${ }^{1,2 \star ~ J a c o p o ~ F r i t z, ~}{ }^{3}$ Itziar Aretxaga, ${ }^{1}$ Yalia D. Mayya, ${ }^{1}$ \\ Daniel Rosa-González, ${ }^{1}$ John E. Beckman, ${ }^{4,5,6}$ Gustavo Bruzual ${ }^{3}$ and Stephane Charlot $^{7}$ \\ ${ }^{1}$ Instituto Nacional de Astrofísica, Óptica y Electrónica (INAOE), Luis E. Erro 1, Tonantzintla, Puebla, C.P. 72840, Mexico \\ ${ }^{2}$ Consejo Nacional de Ciencia y Tecnología, Av. Insurgentes Sur 1582, 03940, Ciudad de México, Mexico \\ ${ }^{3}$ Instituto de Radioastronomía y Astrofísica, UNAM, Campus Morelia, 58089 Morelia, Mexico \\ ${ }^{4}$ Instituto de Astrofísica de Canarias, C/ Vía Láctea s/n, 38205 La Laguna, Tenerife, Spain \\ ${ }^{5}$ Department of Astrophysics, University of La Laguna, E-38200 La Laguna, Tenerife, Spain \\ ${ }^{6}$ CSIC, 28006 Madrid, Spain \\ ${ }^{7}$ Sorbonne Université, CNRS, UMR7095, Institut d'Astrophysique de Paris, F-75014, Paris, France
}

Accepted XXX. Received YYY; in original form ZZZ

\begin{abstract}
We have applied stellar population synthesis to $500 \mathrm{pc}$ sized regions in a sample of 102 galaxy discs observed with the MUSE spectrograph. We derived the star formation history and analyse specifically the "recent" (20Myr) and "past" (570Myr) age bins. Using a star formation self-regulator model we can derive local mass-loading factors, $\eta$ for specific regions, and find that this factor depends on the local stellar mass surface density, $\Sigma_{*}$, in agreement with the predictions form hydrodynamical simulations including supernova feedback. We integrate the local $\eta-\Sigma_{*}$ relation using the stellar mass surface density profiles from the Spitzer Survey of Stellar Structure in Galaxies $(\mathrm{S} 4 \mathrm{G})$ to derive global mass-loading factors, $\eta_{\mathrm{G}}$, as a function of stellar mass, $M_{*}$. The $\eta_{\mathrm{G}}-M_{*}$ relation found is in very good agreement with hydrodynamical cosmological zoom-in galaxy simulations. The method developed here offers a powerful way of testing different implementations of stellar feedback, to check on how realistic are their predictions.
\end{abstract}

Key words: galaxies: evolution - galaxies: formation - galaxies: star formation galaxies: stellar content

\section{INTRODUCTION}

Understanding the global star formation process in galaxies is of key importance in the comprehension of galaxy formation and evolution. One of the biggest challenges faced by numerical models of galaxy formation derived directly from cosmological models is to explain why the stellar masses of galaxies are consistently lower than those expected from the simulations (Silk \& Mamon 2012). This difference has been bridged by invoking internal mechanisms capable of regulating the star formation rate. Two regimes have been generally used: for massive galaxies their nuclear activity is found to be a mechanism which acts in this way (Martín-Navarro et al. 2018). But for low mass galaxies the star formation itself, through feedback, appears to offer a satisfactory mechanism to reduce the star formation rate, making star formation an inefficient process when comparing the stars which

^ E-mail: javier.zaragoza@inaoep.mx are formed with the availability of gas to form them (Bigiel et al. 2008; Hopkins et al. 2014; Kruijssen et al. 2019). Star formation self-regulates by expelling gas, and the amount of gas that flows out of any system is considered to depend on the mass of stars formed.

The models used to explain the consistently low mean star formation rate (SFR) efficiency use sub-grid physics parametrised by a mass loading factor, $\eta$, relating the mass outflow rate $\dot{M}_{\text {out }}$ and the SFR by $\dot{M}_{\text {out }}=\eta$ SFR (Schaye et al. 2010; Vogelsberger et al. 2013; Somerville \& Davé 2015; Hopkins et al. 2018).

This factor can be predicted by modelling the feedback process (Creasey et al. 2013; Muratov et al. 2015; Li et al. 2017), or inferred from observations (Schroetter et al. 2019; McQuinn et al. 2019; Kruijssen et al. 2019; Roberts-Borsani et al. 2020). However feedback modelling has many uncertainties, and the required observations are scarce and also subject to uncertainty. The present article marks a significant step in making up for the observational deficiencies. 
In order to see whether the star formation at different epochs is correlated and to quantify it by estimating the mass-loading factor we apply an empirical method based on stellar population synthesis and the self-regulator model of star formation, which has been presented previously (Zaragoza-Cardiel et al. 2019).

The star formation self-regulator model (Bouché et al. 2010; Lilly et al. 2013; Dekel \& Mandelker 2014; Forbes et al. 2014; Ascasibar et al. 2015) assumes mass conservation for a galaxy, which implies that the change per unit time of the gas mass, $\dot{M}_{\text {gas }}$, equals the inflow rate into the galaxy, $\dot{M}_{\text {in }}$, minus the gas that goes into star formation, SFR, and the gas which flows out of the galaxy, $\dot{M}_{\text {out }}$ :

$\dot{M}_{\mathrm{gas}}=\dot{M}_{\mathrm{in}}-\operatorname{SFR}(1-\mathrm{R}+\eta)$,

where $R$ is the fraction of the mass which is returned to the interstellar medium from the stellar population.

The spatially resolved star formation self-regulator model applies to segments of a galaxy (Zaragoza-Cardiel et al. 2019), where by segment we mean any spatially resolved region of a galaxy. In these resolved regions we also assume conservation of mass: the time change of the gas mass surface density in a segment, $\dot{\Sigma}_{\text {gas }}$, is equal to the surface density of the net gas flow rate, $\dot{\Sigma}_{\text {net flow, }}$, minus the surface density of gas that goes into new stars through star formation, $\Sigma_{\mathrm{SFR}}$, and minus the surface density of gas that is expelled from the segment by stellar processes, $\dot{\Sigma}_{\text {out }}$ :

$\dot{\Sigma}_{\text {gas }}=\dot{\Sigma}_{\text {net flow }}-\Sigma_{\mathrm{SFR}}(1-R+\eta)$

where $R$ is the fraction of the mass that is returned to the interstellar medium, and

$\dot{\Sigma}_{\text {out }}=\eta \Sigma_{\text {SFR }}$.

This model allows us to relate the star formation rate surface density in a segment, $\Sigma_{\mathrm{SFR}}$, to the change in gas mass in that segment. The complex processes of stellar feedback are parameterized by the mass-loading factor: $\dot{\Sigma}_{\text {out }}=\eta \Sigma_{\mathrm{SFR}}$.

We present the galaxy sample and the data in section $\S 2$. In section $\S 3$ we give the stellar population synthesis fits and also fit the observables to the star formation selfregulator model. In section $\S 4$ we show the results obtained, and the variation of $\eta$, while in section $\S 5$ we convert local values of $\eta$ into global ones. We discuss our results in section $\S 6$ and present our conclusions in section $\S 7$.

\section{GALAXY SAMPLE AND DATA}

\subsection{Galaxy sample}

A significant number of galaxies have been observed with the MUSE instrument on the VLT in different surveys (Poggianti et al. 2017; Sánchez-Menguiano et al. 2018; Kreckel et al. 2019; Erroz-Ferrer et al. 2019; López-Cobá et al. 2020). To use these observations we build our sample using the Hyperleda database and looking for them in the MUSE archive. To be able to apply the method for a given galaxy, we need to resolve the galaxy at a specific spatial scale. Based on results of NGC 628 (Zaragoza-Cardiel et al. 2019), we choose the $500 \mathrm{pc}$ scale to study the star formation self-regulation so we are limited to galaxies closer than $100 \mathrm{Mpc}$ to resolve 500 pc at 1 arcsec resolution. We also need enough $(\sim 16)$ resolution elements, so very nearby galaxies with low number of 500pc resolution elements are not useful. We will divide the MUSE field of view in squares, so we will need at least $4 \times 4500$ pc squares per galaxy, limiting us to galaxies further away than $7 \mathrm{Mpc}$.

We need galaxies with recent star formation to study star formation self-regulation. To ensure that we will detect recent star formation, we just consider Sa or later types morphology (Hubble type $T \geq 1.0$ in Hyperleda). We discard edge-on galaxies $(i=90 \mathrm{deg})$, galaxies classified as multiple, Irregulars (Hubble type $T \geq 9$ in Hyperleda), and LIRGs (in NASA Ned). We just select galaxies with declination lower than $45 \mathrm{degN}$ to be observable from Paranal Observatory.

The SQL (Structured Query Language) search through Hyperleda ${ }^{1}$ selects 13636 galaxies, of which 164 have been observed with MUSE on the VLT and have publicly available data with an exposure time at least of 1600 seconds. We also removed galaxies in Arp (Arp 1966), Vorontsov-Velyaminov (Vorontsov-Velyaminov 1959), and Hickson Compact Group (Hickson 1982) catalogs, to get rid out of strong external effects on the star formation history $(\mathrm{SFH})$ and gas flows due to interactions. We have a total of 148 galaxies satisfying these conditions in the public MUSE archive. Of these, 9 galaxies did not pass our requirements in a spectral inspection by eye, because of clear spectral artifacts, or not having enough $\mathrm{H} \alpha$ emission in the pointing (MUSE has a square 1 arcmin $\times 1$ arcmin FOV). We initially analysed the single stellar populations (SSP's) of the remaining 139 galaxies, to apply the method described in this article. Since the method requires enough regions to be include in the analysis, we set this limit to $16(4 \times 4)$. However each of the 16 regions sampled per galaxy needs sufficient current SFR, sufficient signal to noise, and that can be properly reproduced with stellar population synthesis models. Finally, only 102 galaxies satisfied all the conditions allowing us to estimate $\eta$. We present their parameters in Table 1.

\subsection{Muse spectral data}

We use the MUSE (Bacon et al. 2010) reduced publicly available data for the galaxies listed in Table 1, from the ESO archive $^{2}$.

We first made a visual inspection to remove galaxies with no $\mathrm{H} \alpha$ emission in the MUSE pointing, and thus to select MUSE fields where $\mathrm{H} \alpha$ was observed, to be able to estimate recent star formation. After delimiting the regions with recent star formation, we divide each field into an integer number of observing squares, giving us squares with the closest (and larger than) size value to $500 \mathrm{pc}$. We show an example in Fig. 1, we do not use the squares outside the MUSE pointing. We choose $500 \mathrm{pc}$ because it gives us a scale on which, from previous work, we expect to observe the selfregulation of star formation (Zaragoza-Cardiel et al. 2019), and it allows us to include galaxies at distances of up to 100

\footnotetext{
1 http://leda.univ-lyon1.fr/fullsql.html

2 http://archive.eso.org/wdb/wdb/adp/phase3_spectral/ form?collection_name=MUSE
} 
Table 1. Galaxy sample.

\begin{tabular}{|c|c|c|c|c|c|}
\hline Galaxy identifier & PGC identifier $^{a}$ & $\begin{array}{c}D^{b} \\
\mathrm{Mpc}\end{array}$ & $\mathrm{z}^{c}$ & Type $^{d}$ & $\mathrm{i}^{e}$ \\
\hline pgc33816 & PGC33816 & 23.6 & 0.005187 & 7.0 & 19.9 \\
\hline eso184-g082 & PGC63387 & 35.2 & 0.00867 & 4.1 & 32.6 \\
\hline eso467-062 & PGC68883 & 57.5 & 0.013526 & 8.6 & 49.9 \\
\hline ugc 272 & PGC1713 & 55.6 & 0.012993 & 6.5 & 70.7 \\
\hline ngc5584 & PGC51344 & 23.1 & 0.005464 & 5.9 & 42.4 \\
\hline eso319-g015 & PGC34856 & 37.5 & 0.009159 & 8.6 & 54.2 \\
\hline ugc11214 & PGC61802 & 38.0 & 0.008903 & 5.9 & 16.5 \\
\hline ngc6118 & PGC57924 & 20.5 & 0.005247 & 6.0 & 68.7 \\
\hline ic1158 & PGC56723 & 24.5 & 0.006428 & 5.1 & 62.2 \\
\hline ngc5468 & PGC50323 & 30.0 & 0.00948 & 6.0 & 21.1 \\
\hline eso325-g045 & PGC50052 & 75.9 & 0.017842 & 7.0 & 40.2 \\
\hline ngc1954 & PGC17422 & 38.0 & 0.010441 & 4.4 & 61.5 \\
\hline ic5332 & PGC71775 & 9.9 & 0.002338 & 6.8 & 18.6 \\
\hline ugc04729 & PGC25309 & 57.0 & 0.013009 & 6.0 & 35.2 \\
\hline ngc2104 & PGC17822 & 16.4 & 0.003873 & 8.5 & 83.6 \\
\hline eso316-g7 & PGC28744 & 47.5 & 0.01166 & 3.3 & 70.0 \\
\hline eso298-g28 & PGC8871 & 70.1 & 0.016895 & 3.8 & 64.4 \\
\hline mcg-01-57-021 & PGC69448 & 30.6 & 0.009907 & 4.0 & 52.2 \\
\hline pgc128348 & PGC128348 & 61.1 & 0.014827 & 5.0 & 36.7 \\
\hline pgc1167400 & PGC1167400 & 60.0 & 0.01334 & 4.0 & 30.5 \\
\hline ngc 2835 & PGC26259 & 10.1 & 0.002955 & 5.0 & 56.2 \\
\hline ic2151 & PGC18040 & 30.6 & 0.010377 & 3.9 & 61.5 \\
\hline ngc988 & PGC9843 & 17.3 & 0.005037 & 5.9 & 69.1 \\
\hline ngc1483 & PGC14022 & 16.8 & 0.003833 & 4.0 & 37.3 \\
\hline ngc7421 & PGC70083 & 24.2 & 0.005979 & 3.7 & 36.2 \\
\hline fcc 290 & PGC13687 & 19.0 & 0.004627 & 2.1 & 48.1 \\
\hline ic344 & PGC13568 & 75.6 & 0.018146 & 4.0 & 60.7 \\
\hline ngc3389 & PGC32306 & 21.4 & 0.004364 & 5.3 & 66.2 \\
\hline eso246-g21 & PGC9544 & 76.6 & 0.018513 & 3.0 & 52.4 \\
\hline pgc170248 & PGC170248 & 85.1 & 0.019163 & 4.7 & 76.4 \\
\hline ngc7329 & PGC69453 & 45.7 & 0.010847 & 3.6 & 42.7 \\
\hline ugc12859 & PGC72995 & 78.3 & 0.018029 & 4.0 & 72.8 \\
\hline ugc1395 & PGC7164 & 74.1 & 0.017405 & 3.1 & 55.1 \\
\hline ngc5339 & PGC49388 & 27.0 & 0.009126 & 1.3 & 37.5 \\
\hline ngc1591 & PGC15276 & 55.8 & 0.013719 & 2.0 & 56.8 \\
\hline pgc98793 & PGC98793 & 55.2 & 0.01292 & 5.0 & 0.0 \\
\hline ugc5378 & PGC28949 & 56.5 & 0.01388 & 3.1 & 64.1 \\
\hline ngc4806 & PGC44116 & 29.0 & 0.008032 & 4.9 & 32.9 \\
\hline ngc1087 & PGC10496 & 14.4 & 0.00506 & 5.2 & 54.1 \\
\hline ngc4980 & PGC45596 & 16.9 & 0.004767 & 1.1 & 71.5 \\
\hline ngc6902 & PGC64632 & 46.6 & 0.009326 & 2.3 & 40.2 \\
\hline ugc11001 & PGC60957 & 63.3 & 0.01406 & 8.1 & 78.7 \\
\hline ic217 & PGC8673 & 27.0 & 0.006304 & 5.8 & 82.6 \\
\hline eso506-g004 & PGC39991 & 57.5 & 0.013416 & 2.6 & 67.2 \\
\hline ic 2160 & PGC18092 & 64.7 & 0.015809 & 4.6 & 62.7 \\
\hline ngc1385 & PGC13368 & 22.7 & 0.005 & 5.9 & 52.3 \\
\hline mcg-01-33-034 & PGC43690 & 32.0 & 0.008526 & 2.1 & 56.6 \\
\hline
\end{tabular}

${ }^{a}$ Principal General Catalog of Galaxies identifier from Hyperleda database (Paturel et al. 2003). ${ }^{b}$ Distance from the $\mathrm{z}=0$ Multi-wavelength Galaxy Synthesis (z0MGS from Leroy et al. (2019), when available) and HyperLeda database best homogenized distances (Makarov et al. 2014). ${ }^{c}$ Redshift, from Nasa Ned. ${ }^{d}$ Numerical morphologycal type, from the HyperLeda database. ${ }^{e}$ Inclination from the HyperLeda database.

Mpc where 500 pc corresponds to 1 arcsec. We also need the foreground stars to be masked. We extract the spectrum for each defined region, correct it for Galactic extinction, and associate each with a redshift estimate, using the $\mathrm{H} \alpha$ or $[\mathrm{NII}]$ at 6583.4 if the later has a stronger peak than the former. We next estimate the $[\mathrm{NII}] / \mathrm{H} \alpha$ and the $[\mathrm{OIII}] / \mathrm{H} \beta$ flux ratios, and remove the regions which are classified as Seyfert-LINER in the BPT diagram (Kewley et al. 2006). 
Table 1 - continued

\begin{tabular}{|c|c|c|c|c|c|}
\hline Galaxy identifier & PGC identifier $^{a}$ & $\begin{array}{l}D^{b} \\
\mathrm{Mpc}\end{array}$ & $\mathrm{z}^{c}$ & Type $^{d}$ & $\mathrm{i}^{e}$ \\
\hline ngc4603 & PGC42510 & 33.1 & 0.008647 & 5.0 & 44.8 \\
\hline ngc4535 & PGC41812 & 15.8 & 0.006551 & 5.0 & 23.8 \\
\hline ngc1762 & PGC16654 & 76.5 & 0.015854 & 5.1 & 51.5 \\
\hline ngc3451 & PGC32754 & 26.1 & 0.00445 & 6.5 & 62.7 \\
\hline ngc4790 & PGC43972 & 15.3 & 0.004483 & 4.8 & 58.8 \\
\hline ngc3244 & PGC30594 & 42.7 & 0.009211 & 5.6 & 49.3 \\
\hline ngc628 & PGC5974 & 9.8 & 0.002192 & 5.2 & 19.8 \\
\hline pgc30591 & PGC30591 & 35.5 & 0.006765 & 6.8 & 86.6 \\
\hline ngc5643 & PGC51969 & 11.8 & 0.003999 & 5.0 & 29.6 \\
\hline ngc1309 & PGC12626 & 24.1 & 0.007125 & 3.9 & 21.2 \\
\hline ngc1084 & PGC10464 & 17.3 & 0.004693 & 4.8 & 49.9 \\
\hline ngc7580 & PGC70962 & 65.3 & 0.01479 & 3.0 & 36.5 \\
\hline ngc692 & PGC6642 & 87.9 & 0.021181 & 4.1 & 45.2 \\
\hline eso462-g009 & PGC64537 & 83.2 & 0.019277 & 1.1 & 58.8 \\
\hline ic5273 & PGC70184 & 14.7 & 0.004312 & 5.6 & 50.8 \\
\hline pgc3140 & PGC3140 & 81.3 & 0.019029 & 1.4 & 62.7 \\
\hline ic 1553 & PGC1977 & 35.0 & 0.00979 & 7.0 & 78.6 \\
\hline ugc11289 & PGC62097 & 59.7 & 0.013333 & 4.5 & 53.7 \\
\hline ic4582 & PGC55967 & 37.3 & 0.007155 & 3.8 & 83.1 \\
\hline ngc2466 & PGC21714 & 73.1 & 0.017722 & 5.0 & 16.0 \\
\hline eso443-21 & PGC44663 & 41.9 & 0.009404 & 5.7 & 79.0 \\
\hline ic4452 & PGC51951 & 65.3 & 0.014337 & 1.3 & 20.6 \\
\hline eso498-g5 & PGC26671 & 40.7 & 0.008049 & 4.3 & 41.8 \\
\hline eso552-g40 & PGC16465 & 95.5 & 0.022649 & 2.1 & 54.4 \\
\hline eso163-g11 & PGC21453 & 33.0 & 0.009413 & 3.0 & 70.9 \\
\hline ngc7582 & PGC71001 & 18.7 & 0.005254 & 2.1 & 68.0 \\
\hline ngc1620 & PGC15638 & 39.6 & 0.011715 & 4.5 & 81.2 \\
\hline ic1320 & PGC64685 & 73.6 & 0.016548 & 2.9 & 58.1 \\
\hline ngc3393 & PGC32300 & 52.8 & 0.012509 & 1.2 & 30.9 \\
\hline ngc2370 & PGC20955 & 79.8 & 0.018346 & 3.4 & 56.8 \\
\hline ngc4981 & PGC45574 & 21.0 & 0.005604 & 4.0 & 44.7 \\
\hline ngc3783 & PGC36101 & 25.1 & 0.00973 & 1.4 & 26.6 \\
\hline ngc1285 & PGC12259 & 74.1 & 0.017475 & 3.4 & 59.3 \\
\hline ngc5806 & PGC53578 & 26.2 & 0.004533 & 3.2 & 60.4 \\
\hline eso018-g018 & PGC26840 & 71.1 & 0.017572 & 4.2 & 38.9 \\
\hline ngc6754 & PGC62871 & 38.4 & 0.010864 & 3.2 & 61.0 \\
\hline ic 2560 & PGC29993 & 32.5 & 0.009757 & 3.4 & 65.6 \\
\hline $\operatorname{ngc} 7140$ & PGC67532 & 36.0 & 0.009947 & 3.8 & 49.6 \\
\hline ngc3464 & PGC833131 & 52.8 & 0.012462 & 4.9 & 50.8 \\
\hline mcg-02-13-38 & PGC16605 & 55.2 & 0.013293 & 1.2 & 73.6 \\
\hline ngc1590 & PGC15368 & 55.2 & 0.012999 & 5.0 & 27.9 \\
\hline pgc 8822 & PGC8822 & 74.1 & 0.017555 & 5.0 & 58.2 \\
\hline ngc7721 & PGC72001 & 21.2 & 0.006721 & 4.9 & 81.4 \\
\hline $\operatorname{pgc} 28308$ & PGC28308 & 43.0 & 0.00907 & 6.7 & 85.5 \\
\hline ngc1137 & PGC10942 & 42.8 & 0.010147 & 3.0 & 59.5 \\
\hline eso478-g006 & PGC8223 & 74.8 & 0.017786 & 4.2 & 57.7 \\
\hline ngc1448 & PGC13727 & 16.8 & 0.003896 & 6.0 & 86.4 \\
\hline ngc3278 & PGC31068 & 42.7 & 0.009877 & 5.1 & 41.0 \\
\hline ngc4030 & PGC37845 & 19.0 & 0.004887 & 4.0 & 47.0 \\
\hline ngc3363 & PGC32089 & 85.1 & 0.019233 & 3.5 & 45.3 \\
\hline ngc7780 & PGC72775 & 76.2 & 0.017195 & 2.0 & 61.2 \\
\hline ic1438 & PGC68469 & 42.5 & 0.008659 & 1.2 & 23.8 \\
\hline ngc4666 & PGC42975 & 15.7 & 0.005101 & 5.0 & 69.6 \\
\hline ngc7396 & PGC69889 & 71.8 & 0.016561 & 1.0 & 59.5 \\
\hline ngc716 & PGC6982 & 65.9 & 0.015204 & 1.1 & 75.9 \\
\hline
\end{tabular}

\section{STELLAR POPULATION SYNTHESIS AND MODEL FITS}

\subsection{Stellar population synthesis}

We use SINOPSIS code ${ }^{3}$ (Fritz et al. 2007, 2017) to fit combinations of SSPs to the observed spectra. SINOPSIS fits equivalent widths of emission and absorption lines, as well as defined continuum bands. In this work, we use the $\mathrm{H} \alpha$ and $\mathrm{H} \beta$ equivalent widths, and the 9 continuum bands shown in Fig. 2, where we show two observed spectra of the galaxy NGC 716 and the resulted fits as an example. 


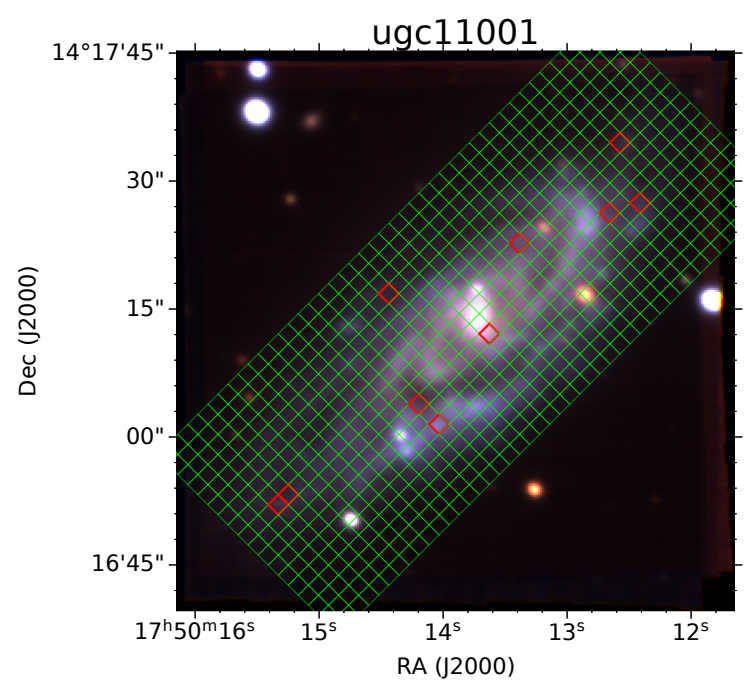

Figure 1. Colour composite RGB image recovered from MUSE data of one of the studied galaxies, UGC 11001. The red, green and blue images used to create RGB are obtained by integrating MUSE spectra in R, V and B filters, respectively. 500pc wide regions where spectra was extracted are overplotted as green and the regions identified as those on the envelope (defined above in section $\S 4)$ are marked as red squares.

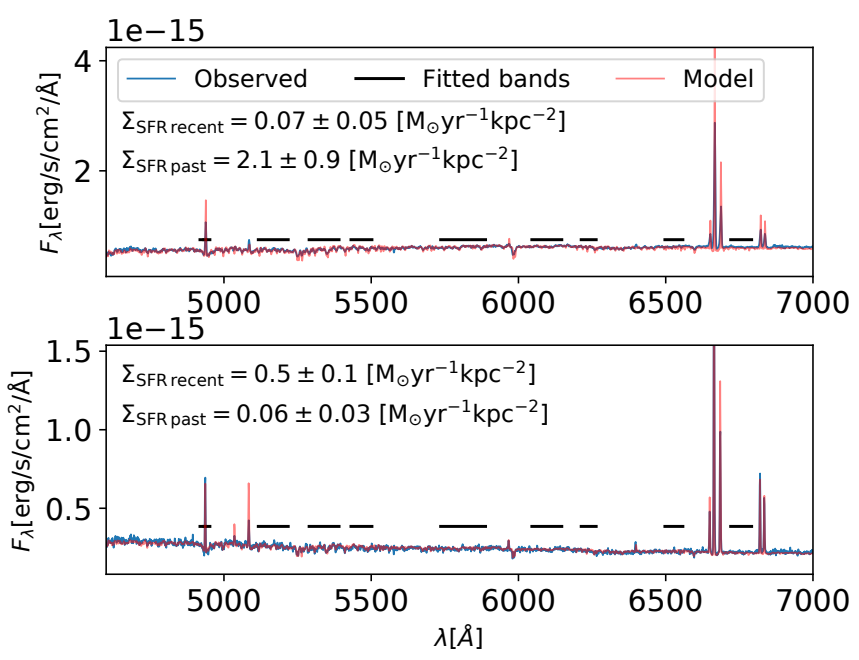

Figure 2. Two characteristic spectra for the galaxy NGC 716 . Observed spectra are shown as blue lines. Top: the total star formation is dominated by the past star formation. Bottom: the total star formation is dominated by the recent star formation. The model spectrum which best fits the observed spectrum is shown as a red line. The continuum bands used to fit the observed spectrum to the combination of SSPs are shown as black lines.

We use the updated version of the Bruzual \& Charlot models (Werle et al. 2019). We used SSPs of 3 metallicities $(Z=0.004, Z=0.02$, and $Z=0.04)$ in 12 age bins ( $2 \mathrm{Myr}$, $4 \mathrm{Myr}, 7 \mathrm{Myr}, 20 \mathrm{Myr}, 57 \mathrm{Myr}, 200 \mathrm{Myr}, 570 \mathrm{Myr}, 1 \mathrm{Gyr}$, $3 \mathrm{Gyr}, 5.75 \mathrm{Gyr}, 10 \mathrm{Gyr}$, and $14 \mathrm{Gyr}$ ). We assume a free form of SFH, the Calzetti dust attenuation law (Calzetti et al. 2000), and the Chabrier 2003 IMF (Chabrier 2003) for stellar masses between $0.1 \mathrm{M}_{\odot}$ and $100 \mathrm{M}_{\odot}$. The emission lines for the SSPs younger than 20 Myr are computed using the photoionisation code CLOUDY (Ferland 1993; Ferland et al. 1998, 2013), assuming case B recombination (Osterbrock 1989), an electron temperature of $10^{4} \mathrm{~K}$, an electron density of $100 \mathrm{~cm}^{-3}$, and a gas cloud with an inner radius of $10^{-2} \mathrm{pc}$ (Fritz et al. 2017).

SINOPSIS uses the degeneracies between age, metallicity, and dust attenuation, to compute the uncertainties in the derived parameters (Fritz et al. 2007).

We rebin the different age bins into 4 bins: at 20Myr, $570 \mathrm{Myr}, 5.7 \mathrm{Gyr}$, and $14 \mathrm{Gyr}$. Simulated and observed spectra have been used to prove the validity of using SINOPSIS to recover these 4 age bins (Fritz et al. 2007, 2011). Additionally, SINOPSIS and similar codes have shown the reliability of recovering the SFH using synthesis of SSPs in, at least, 4 age bins (Cid Fernandes et al. 2005; Fritz et al. 2007, 2011; Sánchez et al. 2016). However, since we are interested in the recent star formation variations, we consider only the two most recent age bins, $20 \mathrm{Myr}, 570 \mathrm{Myr}$, and call them recent, and past age bins, respectively. In this way we recover the recent, and the past star formation rate surface densities, $\Sigma_{\text {SFR recent }}$ and $\Sigma_{\text {SFR past }}$, which improves the confidence in the results presented in this work, since the two most recent age bins are better constrained than the oldest ones.

In order to use regions with a meaningful result, we take into account only regions with a signal to noise ratio (SNR) larger than 20 over the [5350-5420] range, and $\chi^{2}<3$. Due to IMF sampling effects, we also consider only regions where the recent SFR is larger than $10^{-3} \mathrm{M}_{\odot} / \mathrm{yr}$ and the past SFR is larger than $10^{-5} \mathrm{M}_{\odot} / \mathrm{yr}$.

Because we are limited to galaxies from Hubble Type Sa to Sdm, also excluding interacting galaxies and (U)LIRGs, the galaxy sample, by construction, is defined by galaxies that are probably on the star formation galaxy main sequence, and probably evolved via secular evolution in the studied age range (last $570 \mathrm{Myr}$ ), where by secular evolution we mean evolution dominated by slow processes (slower than many galaxy rotation periods Kormendy \& Kennicutt (2004)). The galaxies probably evolved through more violent episodes in the past, but we are not affected by them in the studied age range. Nevertheless, individual zones such as the centres of the galaxies, might have evolved via rapid evolution due to high gas flows even in the studied age range. Because of this, we removed regions whose centres are at a distance of $500 \mathrm{pc}$ or less from the centre of the galaxy, as well as regions having a very high recent SFR compared to the rest of the galaxy, specifically, we removed regions having $\Sigma_{\text {SFR recent larger than }} \bar{\Sigma}_{\text {SFR recent }}+3 \sigma_{\Sigma_{\text {SFR recent }}}$ for each galaxy. We will discuss how affects the results the removal of very high recent SFR regions in the discussion section (§6.4).

\subsection{Fitting data to self-regulator the model}

We have made the same assumptions made in ZaragozaCardiel et al. (2019) in order to fit our observables to the self-regulator model. For completeness, we briefly describe them here.

The self-regulator model (Eq. 2) is valid for a star or a group of co-rotating stars in the galaxy such as a massive star cluster $\left(>500 \mathrm{M}_{\odot}\right.$ Lada \& Lada (2003)). Assuming $\eta$ constant, Eq. 2 is linear, so we can add up regions obeying that equation, and still obey the equation. In this context, the 
mass-loading factor would be representative of massive star clusters scales ( pc Lada \& Lada (2003)). Although we find below that $\eta$ varies (Eq. 6), the variation is smooth enough to consider it approximately constant here. Therefore, the group of stars which are massive enough to produce bound clusters can be considered as a whole, while the less massive ones are splitted into individual stars. Feedback between different regions is then not considered here. We assume that our $500 \mathrm{pc}$ wide regions are made of individual smaller regions obeying Eq 2, so we can rewrite Eq. 2 to be valid for our larger regions as the average of individual regions:

$$
\dot{\bar{\Sigma}}_{\text {gas }}=\dot{\bar{\Sigma}}_{\text {net flow }}-\bar{\Sigma}_{\text {SFR }}(1-R+\eta)
$$

We already showed in Zaragoza-Cardiel et al. (2019) that the resulted mass-loading factor was independent of the chosen scale (from $87 \mathrm{pc}$ to $1 \mathrm{kpc}$ ) in NGC 628. Hence, regions can be added up while Eq. 4 is still valid.

The value of the $\bar{\Sigma}_{\text {SFR past }}$ we are able to measure is a time average over $550 \mathrm{Myr}$. Since Eq. 4 is linear, we can substitute the time differentials by time average values over our age bin, and we will not be affected by possible bursts of the star formation, as long as the variation of $\eta$ is small enough (as we do find below).

The net gas flow rate surface density, $\dot{\bar{\Sigma}}_{\text {net flow, }}$ is the change in gas density due to gas flows (independently of star formation), which can be negative, although in that case, the star formation is quenched (Zaragoza-Cardiel et al. 2019). This term, $\dot{\bar{\Sigma}}_{\text {net flow }}$, also includes the possibility of gas return from different regions and the same region at a later epoque, an effect known as galactic fountains (Fraternali 2017). The observables are $\bar{\Sigma}_{\text {SFR recent }}$ and $\bar{\Sigma}_{\text {SFR past }}$. Let us assume that we can estimate $\dot{\bar{\Sigma}}_{\text {gas }}$ from the star formation change considering the KS law, $\bar{\Sigma}_{\mathrm{SFR}}=A \bar{\Sigma}_{\text {gas }}^{N}$, and rewrite Eq. 2 :

$$
\begin{aligned}
& \bar{\Sigma}_{\text {SFR recent }}= \\
& A\left\{\left[\dot{\bar{\Sigma}}_{\text {net flow }}-\bar{\Sigma}_{\text {SFR past }}(1-R+\eta)\right] \Delta t+\left[\frac{\bar{\Sigma}_{\text {SFR past }}}{A}\right]^{\frac{1}{N}}\right\}^{N} .
\end{aligned}
$$

where there is a relation between our two observables $\left(\bar{\Sigma}_{\text {SFR recent }}\right.$ and $\left.\bar{\Sigma}_{\text {SFR past }}\right), \dot{\bar{\Sigma}}_{\text {net flow }}$, and $\eta$. We will use a simplistic approximation to estimate $\dot{\bar{\Sigma}}_{\text {net flow }}$, since we do not observe it. As explained in Zaragoza-Cardiel et al. (2019), we assume that several regions have an approximate value close to the maximum value of $\dot{\bar{\Sigma}}_{\text {net flow }}$, for a given galaxy. In the case of the estimation of $\eta$, although $\eta$ could vary between regions, we will find that the variation is smooth enough (Eq. 6) to consider the existence of a representative value for specific regions. In the following, for simplicity since we are only dealing with one type of regions, the $500 \mathrm{pc}$ wide ones, we will be using the analysed terms (e.g. $\bar{\Sigma}_{\text {SFR recent }}$, $\left.\bar{\Sigma}_{\text {SFR past }}, \dot{\bar{\Sigma}}_{\text {net flow }}\right)$ without the need of using the average symbols $\left(\Sigma_{\text {SFR recent }}, \Sigma_{\text {SFR past }}, \dot{\Sigma}_{\text {net flow }}\right)$. Therefore, when we present an average, the average will be for several 500pc wide regions.

Assuming the instantaneous recycling approximation (Madau \& Dickinson 2014) for stars more massive than $3 \mathrm{M}_{\odot}$ ( $\tau_{\mathrm{MS}} \sim 0.6 \mathrm{Gyr}$, where $\tau_{\mathrm{MS}}$ is the main sequence lifetime), and a Chabrier IMF (Chabrier 2003), we obtain a value of

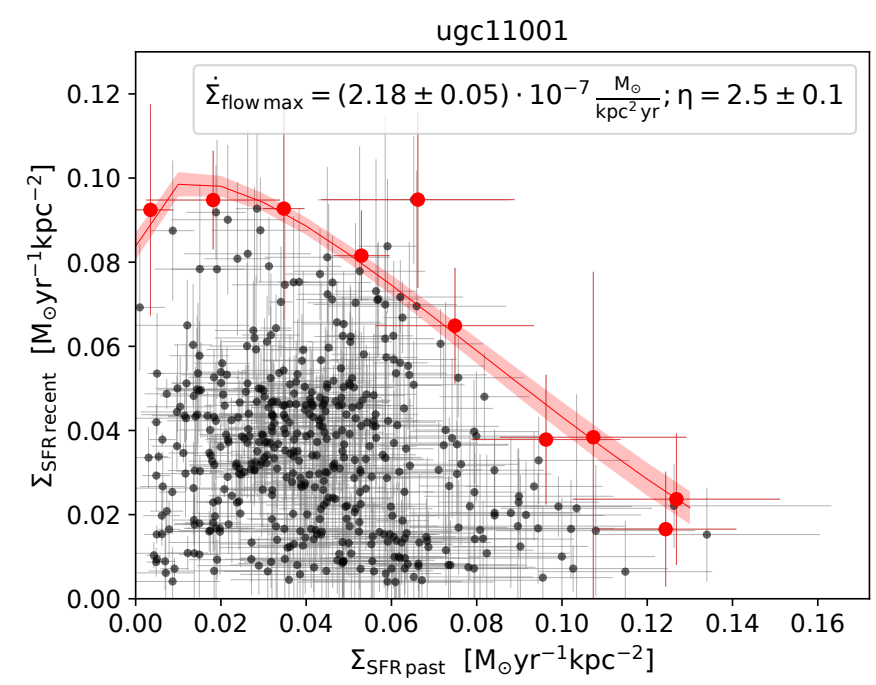

Figure 3. Recent star formation rate surface density, $\Sigma_{\text {SFR recent }}$, versus the past star formation rate surface density, $\Sigma_{\text {SFR past }}$, for the UGC 11001 galaxy. The red dots are the regions identified as those on the envelope. We plot the fit of Eq. 5 to the regions on the envelope as well as the result of the fit and the $1-\sigma$ uncertainty range of the fit as shaded region.

$R=0.27$. We use the values $A=10^{-4.32} \mathrm{M}_{\odot} / \mathrm{kpc}^{2} / \mathrm{yr}$, and $N=1.56$ for the KS lawKennicutt et al. (2007).

\section{RESULTS}

As an example, we plot the $\Sigma_{\text {SFR recent versus }} \Sigma_{\text {SFR past }}$ diagram for one of the galaxies, UGC 11001, in Fig. 3. We plot the $\Sigma_{\text {SFR recent versus }} \Sigma_{\text {SFR past }}$ diagrams for all of the galaxies in Fig. A1. Each of the points in these plots can be seen as the relation between the $\Sigma_{\text {SFR recent }}$ and the $\Sigma_{\text {SFR past }}$ which depends on the value of $\dot{\Sigma}_{\text {net flow }}$, and $\eta$ (Eq. 5).

We identify those regions having the maximum $\Sigma_{\text {SFR recent }}$, per bin of $\Sigma_{\text {SFR past }}$, as the regions on the envelope. We see the regions on the envelope as red dots in Fig. 3 , and as red squares in the MUSE recovered false color image of UGC 11001 in Fig. 1.

We can see in Eq. 5 that we need the value of $\dot{\Sigma}_{\text {net flow }}$ in combination with the $\Sigma_{\text {SFR recent }}$ and $\Sigma_{\text {SFR past }}$ values, in order to quantify $\eta$. For a given galaxy, there should be a maximum value for the net flow gas surface density term, $\dot{\Sigma}_{\text {flow max }}$. Although in principle $\dot{\Sigma}_{\text {flow max }}$ is unknown for us, if we assume that there are several segments where $\dot{\Sigma}_{\text {net flow }} \sim$ $\dot{\Sigma}_{\text {flow max }}$, then these regions are those on the $\Sigma_{\text {SFR recent versus }}$ $\Sigma_{\text {SFR past }}$ diagram envelope.

Assuming $\dot{\Sigma}_{\text {net flow }}$ constant, we fit Eq. 5 to the regions on the envelope and estimate the mass-loading factor, representative of those specific regions. We have selected a galaxy sample mainly composed by galaxies on the main sequence of star formation, and remove segments having very high $\Sigma_{\text {SFR recent }}$ compared to the rest of the segments of a galaxy. Therefore, the galaxy sample, as well as the segments, have been chosen to assure the $\dot{\Sigma}_{\text {net flow }} \sim \dot{\Sigma}_{\text {flow max }}$ hypothesis for several regions. The regions below the envelope are due, to a greater extent, to regions having a smaller value of 
Table 2. Estimated mass-loading factors, $\eta$, maximum flow gas surface density term, $\dot{\Sigma}_{\text {flow max }}$, and the associated average stellar mass surface density for the regions on the envelope, $\Sigma_{*}$.

\begin{tabular}{|c|c|c|c|}
\hline Galaxy identifier & $\eta^{a}$ & $\begin{array}{r}\dot{\Sigma}_{\text {flow max }}{ }^{b} \\
10^{-8} \mathrm{M}_{\odot} \mathrm{yr}^{-1} \mathrm{kpc}^{-2}\end{array}$ & $\begin{array}{r}\Sigma_{*} c \\
10^{6} \mathrm{M}_{\odot} \mathrm{kpc}^{-2}\end{array}$ \\
\hline $\operatorname{pgc} 33816$ & $4.8 \pm 0.9$ & $4.9 \pm 0.9$ & $27 \pm 15$ \\
\hline eso184-g082 & $5.0 \pm 1.0$ & $8.7 \pm 0.6$ & $49 \pm 20$ \\
\hline eso467-062 & $8.0 \pm 2.0$ & $14.0 \pm 1.0$ & $51 \pm 39$ \\
\hline ugc272 & $3.4 \pm 0.2$ & $6.9 \pm 0.4$ & $57 \pm 44$ \\
\hline ngc5584 & $2.2 \pm 0.4$ & $4.0 \pm 1.0$ & $60 \pm 31$ \\
\hline eso319-g015 & $5.0 \pm 2.0$ & $11.0 \pm 3.0$ & $66 \pm 65$ \\
\hline ugc11214 & $2.6 \pm 0.6$ & $9.0 \pm 2.0$ & $84 \pm 33$ \\
\hline ngc6118 & $2.19 \pm 0.09$ & $2.8 \pm 0.3$ & $90 \pm 51$ \\
\hline ic1158 & $6.8 \pm 0.4$ & $16.3 \pm 0.3$ & $109 \pm 28$ \\
\hline ngc5468 & $2.2 \pm 0.8$ & $23.0 \pm 2.0$ & $113 \pm 66$ \\
\hline eso325-g045 & $1.7 \pm 0.2$ & $12.0 \pm 1.0$ & $121 \pm 57$ \\
\hline ngc1954 & $3.3 \pm 0.5$ & $23.8 \pm 0.8$ & $121 \pm 31$ \\
\hline ic5332 & $3.0 \pm 1.0$ & $12.0 \pm 2.0$ & $120 \pm 100$ \\
\hline ugc04729 & $2.8 \pm 0.6$ & $7.0 \pm 2.0$ & $126 \pm 57$ \\
\hline ngc2104 & $1.7 \pm 0.6$ & $4.0 \pm 2.0$ & $132 \pm 62$ \\
\hline eso316-g7 & $2.0 \pm 1.0$ & $12.0 \pm 6.0$ & $136 \pm 39$ \\
\hline eso298-g28 & $6.0 \pm 0.7$ & $47.0 \pm 2.0$ & $136 \pm 46$ \\
\hline mcg-01-57-021 & $7.0 \pm 1.0$ & $17.0 \pm 2.0$ & $137 \pm 24$ \\
\hline $\operatorname{pgc128348}$ & $2.9 \pm 0.1$ & $11.6 \pm 0.7$ & $140 \pm 92$ \\
\hline pgc1167400 & $2.3 \pm 0.3$ & $4.1 \pm 0.8$ & $141 \pm 78$ \\
\hline ngc 2835 & $2.2 \pm 0.7$ & $7.0 \pm 3.0$ & $144 \pm 40$ \\
\hline ic2151 & $2.0 \pm 0.5$ & $5.0 \pm 3.0$ & $146 \pm 61$ \\
\hline ngc988 & $1.2 \pm 0.4$ & $1.0 \pm 2.0$ & $158 \pm 63$ \\
\hline ngc1483 & $3.0 \pm 2.0$ & $13.0 \pm 5.0$ & $158 \pm 40$ \\
\hline ngc7421 & $1.1 \pm 0.3$ & $1.0 \pm 0.9$ & $167 \pm 78$ \\
\hline fcc290 & $2.0 \pm 0.4$ & $3.0 \pm 1.0$ & $169 \pm 42$ \\
\hline ic344 & $2.5 \pm 0.2$ & $11.0 \pm 1.0$ & $171 \pm 78$ \\
\hline ngc3389 & $4.4 \pm 0.7$ & $32.4 \pm 0.7$ & $190 \pm 130$ \\
\hline eso246-g21 & $2.9 \pm 0.7$ & $7.0 \pm 2.0$ & $188 \pm 67$ \\
\hline pgc170248 & $4.9 \pm 0.7$ & $16.0 \pm 1.0$ & $192 \pm 77$ \\
\hline ngc7329 & $4.1 \pm 0.2$ & $7.8 \pm 0.4$ & $200 \pm 120$ \\
\hline ugc12859 & $2.8 \pm 0.3$ & $5.1 \pm 0.9$ & $202 \pm 90$ \\
\hline ugc1395 & $2.7 \pm 0.3$ & $8.0 \pm 1.0$ & $200 \pm 160$ \\
\hline ngc5339 & $2.9 \pm 0.4$ & $5.0 \pm 1.0$ & $210 \pm 150$ \\
\hline ngc1591 & $2.3 \pm 0.7$ & $19.0 \pm 4.0$ & $212 \pm 93$ \\
\hline pgc98793 & $1.7 \pm 0.1$ & $6.6 \pm 0.8$ & $214 \pm 95$ \\
\hline ugc5378 & $2.7 \pm 0.4$ & $10.0 \pm 1.0$ & $223 \pm 93$ \\
\hline ngc4806 & $1.9 \pm 0.2$ & $10.3 \pm 0.9$ & $230 \pm 170$ \\
\hline ngc1087 & $1.3 \pm 0.3$ & $14.0 \pm 2.0$ & $230 \pm 170$ \\
\hline ngc4980 & $1.8 \pm 0.3$ & $6.6 \pm 0.9$ & $240 \pm 170$ \\
\hline ngc6902 & $2.4 \pm 0.3$ & $8.0 \pm 1.0$ & $240 \pm 13$ \\
\hline ugc11001 & $2.5 \pm 0.1$ & $21.8 \pm 0.5$ & $250 \pm 140$ \\
\hline ic217 & $1.2 \pm 0.4$ & $3.0 \pm 2.0$ & $266 \pm 88$ \\
\hline eso506-g004 & $3.4 \pm 0.2$ & $13.9 \pm 0.4$ & $270 \pm 170$ \\
\hline ic 2160 & $2.4 \pm 0.4$ & $12.0 \pm 2.0$ & $270 \pm 260$ \\
\hline ngc1385 & $0.9 \pm 0.1$ & $9.7 \pm 0.9$ & $272 \pm 44$ \\
\hline mcg-01-33-034 & $1.0 \pm 0.09$ & $7.9 \pm 0.5$ & $270 \pm 150$ \\
\hline ngc4603 & $0.9 \pm 0.1$ & $3.0 \pm 1.0$ & $276 \pm 85$ \\
\hline ngc4535 & $4.1 \pm 0.7$ & $15.0 \pm 2.0$ & $280 \pm 200$ \\
\hline ngc1762 & $2.3 \pm 0.2$ & $8.2 \pm 0.7$ & $290 \pm 140$ \\
\hline ngc3451 & $3.7 \pm 0.4$ & $11.0 \pm 1.0$ & $300 \pm 180$ \\
\hline ngc4790 & $1.2 \pm 0.3$ & $9.0 \pm 2.0$ & $334 \pm 63$ \\
\hline ngc3244 & $1.8 \pm 0.2$ & $8.0 \pm 1.0$ & $340 \pm 270$ \\
\hline ngc628 & $1.8 \pm 0.1$ & $19.0 \pm 1.0$ & $360 \pm 170$ \\
\hline pgc30591 & $0.7 \pm 0.4$ & $0.0 \pm 2.0$ & $360 \pm 160$ \\
\hline ngc5643 & $1.1 \pm 0.3$ & $3.0 \pm 2.0$ & $410 \pm 160$ \\
\hline ngc1309 & $1.3 \pm 0.2$ & $17.0 \pm 2.0$ & $420 \pm 220$ \\
\hline
\end{tabular}

$\bar{a}$ Mass-loading factor derived in this work. ${ }^{b}$ Maximum flow gas surface density term derived in this work. ${ }^{c}$ Stellar mass surface density for the regions on the envelope obtained in this work.
Table 2 - continued

\begin{tabular}{|c|c|c|c|}
\hline Galaxy identifier & $\eta^{a}$ & $\begin{array}{r}\dot{\Sigma}_{\text {flow max }}{ }^{b} \\
10^{-8} \mathrm{M}_{\odot} \mathrm{yr}^{-1} \mathrm{kpc}^{-2}\end{array}$ & $\begin{array}{r}\sum_{*} c \\
10^{6} \mathrm{M}_{\odot} \mathrm{kpc}^{-2}\end{array}$ \\
\hline ngc1084 & $0.6 \pm 0.2$ & $13.0 \pm 6.0$ & $420 \pm 170$ \\
\hline ngc7580 & $1.6 \pm 0.2$ & $18.0 \pm 1.0$ & $420 \pm 110$ \\
\hline ngc692 & $2.7 \pm 0.2$ & $14.0 \pm 1.0$ & $420 \pm 220$ \\
\hline eso462-g009 & $4.0 \pm 1.0$ & $8.0 \pm 2.0$ & $440 \pm 310$ \\
\hline ic5273 & $1.5 \pm 0.7$ & $9.0 \pm 4.0$ & $450 \pm 220$ \\
\hline pgc3140 & $1.0 \pm 0.1$ & $4.0 \pm 1.0$ & $460 \pm 210$ \\
\hline ic1553 & $0.6 \pm 0.3$ & $5.0 \pm 2.0$ & $460 \pm 290$ \\
\hline ugc11289 & $1.8 \pm 0.3$ & $8.0 \pm 3.0$ & $472 \pm 46$ \\
\hline ic4582 & $2.0 \pm 0.1$ & $9.1 \pm 0.5$ & $480 \pm 280$ \\
\hline ngc2466 & $1.5 \pm 0.2$ & $18.0 \pm 2.0$ & $480 \pm 470$ \\
\hline eso443-21 & $1.9 \pm 0.3$ & $94.0 \pm 4.0$ & $490 \pm 180$ \\
\hline ic4452 & $0.37 \pm 0.05$ & $5.2 \pm 0.6$ & $500 \pm 320$ \\
\hline eso498-g5 & $1.5 \pm 0.3$ & $20.0 \pm 2.0$ & $510 \pm 320$ \\
\hline eso552-g40 & $1.7 \pm 0.4$ & $12.0 \pm 3.0$ & $540 \pm 280$ \\
\hline eso163-g11 & $0.48 \pm 0.06$ & $1.0 \pm 2.0$ & $570 \pm 300$ \\
\hline ngc7582 & $0.6 \pm 0.1$ & $0.0 \pm 2.0$ & $570 \pm 280$ \\
\hline ngc1620 & $1.4 \pm 0.3$ & $8.0 \pm 2.0$ & $580 \pm 400$ \\
\hline ic1320 & $1.4 \pm 0.1$ & $4.6 \pm 0.9$ & $590 \pm 270$ \\
\hline ngc3393 & $1.9 \pm 0.3$ & $11.0 \pm 1.0$ & $590 \pm 270$ \\
\hline ngc2370 & $0.87 \pm 0.08$ & $4.0 \pm 2.0$ & $600 \pm 310$ \\
\hline ngc4981 & $0.85 \pm 0.08$ & $7.0 \pm 1.0$ & $630 \pm 580$ \\
\hline ngc3783 & $1.3 \pm 0.3$ & $12.0 \pm 3.0$ & $680 \pm 170$ \\
\hline ngc1285 & $0.8 \pm 0.1$ & $7.0 \pm 2.0$ & $700 \pm 460$ \\
\hline ngc5806 & $1.7 \pm 0.3$ & $17.0 \pm 3.0$ & $720 \pm 530$ \\
\hline eso018-g018 & $1.0 \pm 0.3$ & $13.0 \pm 5.0$ & $720 \pm 420$ \\
\hline ngc6754 & $0.44 \pm 0.06$ & $1.0 \pm 1.0$ & $750 \pm 480$ \\
\hline ic 2560 & $1.2 \pm 0.2$ & $11.0 \pm 1.0$ & $760 \pm 370$ \\
\hline ngc7140 & $2.4 \pm 0.7$ & $13.0 \pm 6.0$ & $770 \pm 210$ \\
\hline ngc3464 & $1.9 \pm 0.3$ & $9.0 \pm 3.0$ & $780 \pm 380$ \\
\hline mcg-02-13-38 & $1.3 \pm 0.1$ & $20.0 \pm 1.0$ & $790 \pm 450$ \\
\hline ngc1590 & $0.83 \pm 0.08$ & $25.0 \pm 3.0$ & $810 \pm 460$ \\
\hline pgc8822 & $1.15 \pm 0.09$ & $4.0 \pm 1.0$ & $970 \pm 430$ \\
\hline ngc7721 & $1.3 \pm 0.5$ & $10 \pm 10$ & $990 \pm 540$ \\
\hline $\operatorname{pgc} 28308$ & $0.2 \pm 0.1$ & $0.0 \pm 8.0$ & $1030 \pm 450$ \\
\hline ngc1137 & $0.8 \pm 0.2$ & $1.0 \pm 4.0$ & $1030 \pm 830$ \\
\hline eso478-g006 & $1.1 \pm 0.2$ & $10.0 \pm 4.0$ & $1050 \pm 650$ \\
\hline ngc1448 & $1.05 \pm 0.09$ & $15.0 \pm 2.0$ & $1130 \pm 480$ \\
\hline ngc3278 & $1.3 \pm 0.2$ & $31.0 \pm 3.0$ & $1170 \pm 710$ \\
\hline ngc4030 & $0.6 \pm 0.1$ & $14.0 \pm 3.0$ & $1310 \pm 550$ \\
\hline ngc3363 & $0.41 \pm 0.08$ & $0.0 \pm 0.9$ & $1330 \pm 590$ \\
\hline ngc7780 & $1.2 \pm 0.2$ & $8.0 \pm 3.0$ & $1450 \pm 590$ \\
\hline ic1438 & $1.5 \pm 0.3$ & $11.0 \pm 4.0$ & $1520 \pm 250$ \\
\hline ngc4666 & $0.3 \pm 0.1$ & $10.0 \pm 3.0$ & $2900 \pm 1300$ \\
\hline ngc7396 & $0.5 \pm 0.2$ & $0.0 \pm 4.0$ & $4100 \pm 1900$ \\
\hline ngc716 & $0.5 \pm 0.2$ & $70 \pm 10$ & $5200 \pm 1900$ \\
\hline
\end{tabular}

$\dot{\Sigma}_{\text {net flow }}<\dot{\Sigma}_{\text {flow max }}$, and to a much lesser extent, to regions having different $\eta$ values.

We have fitted Eq. 5 to the envelopes of the 102 galaxy listed in Table 1 and show the results in Fig. 3 for UGC 11001, and in Fig. A1 for the rest of the galaxies, as a red line.

\subsection{Variations of $\eta$}

Although we have an $\eta$ value for each galaxy, $\eta$ is an average value representative only of the regions on the envelope, instead of the whole galaxy. We associate the estimated $\eta$ for a given envelope with the average surface stellar mass density, $\Sigma_{*}$, of those regions on the envelope where we esti- 


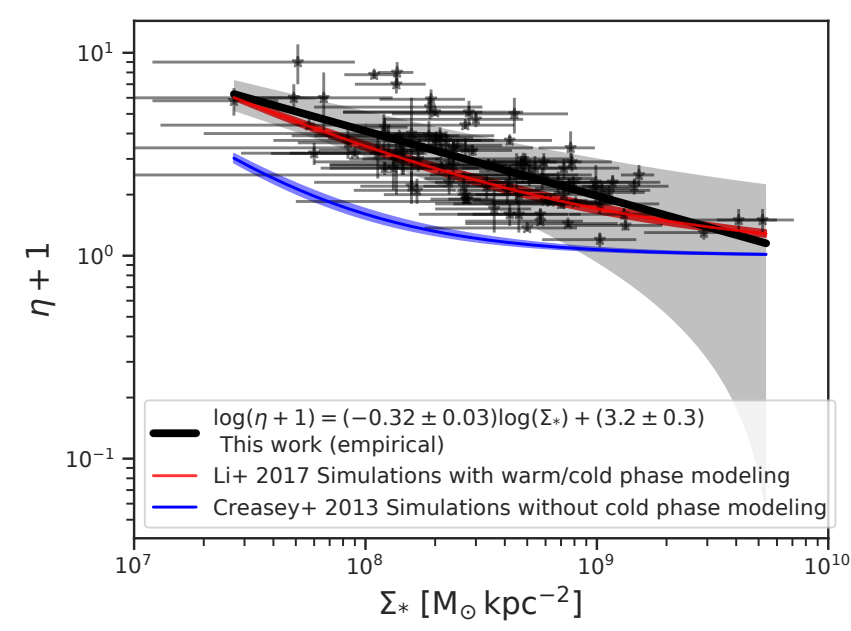

Figure 4. Local mass-loading factor, $\boldsymbol{\eta}$, versus the average stellar mass surface density of the regions on the envelope for each galaxy, $\Sigma_{*}$, as black stars. We plot the linear fit to the empirically derived quantities and the $1-\sigma$ uncertainty range of the fit as shaded regions. The blue line shows the $\eta$ reported for supernova explosions hydrodynamical simulations (Creasey et al. 2013) with cut-off cooling modeling, and as blue line the $\eta$ reported for supernova explosions hydrodynamical simulations (Li et al. 2017) with gas cold-phase modeling included.

mate $\eta$. Therefore, $\eta$ is a local average value, representative only of the regions on the envelope, and their mean value of $\Sigma_{*}$. Although $\eta$ value might vary through the regions on the envelope, we assume that the variation is smooth enough and associate the average stellar mass density, $\Sigma_{*}$ and the standard deviation, to each envelope. The correlation found between $\eta$ and $\Sigma_{*}$ (Eq. 6 and Fig. 4) is in fact smooth enough to make the association between $\eta$ and $\Sigma_{*}$ for the regions on the envelope. We report $\eta, \dot{\Sigma}_{\text {flow max }}$, and $\Sigma_{*}$ values in Table 2 .

We plot in Fig. $4, \eta$ versus $\Sigma_{*}$, and find that the massloading factors strongly correlate with the local $\Sigma_{*}$ measured on the envelope regions:

$\log (\eta+1)=(-0.32 \pm 0.03) \log \left(\Sigma_{*}\right)+(3.2 \pm 0.3)$

This correlation means that the denser the region, the lower is the mass-loading factor, which means that the amount of outflowing gas mass per unit star formation rate depends inversely on the stellar mass surface density. This is so because the denser the region, the larger is the local gravitational pull, making it harder for the gas to be expelled. The local chemical enrichment of galaxies (BarreraBallesteros et al. 2018) also favours the gas regulator model and finds that the mass-loading factor depends on the local escape velocity. This new empirical $\eta$ - $\Sigma_{*}$ relation, can be used to check if stellar feedback implementations in numerical simulations (Hopkins et al. 2014; Li et al. 2017; Creasey et al. 2013) are realistic. In particular, we can compare our $\eta$ $\Sigma_{*}$ relation with the $\eta-\Sigma_{\text {gas }}$ relation from supernova explosion feedback simulations ( $\mathrm{Li}$ et al. 2017; Creasey et al. 2013), using the observed $\Sigma_{*}-\Sigma_{\text {gas }}$ relation (Barrera-Ballesteros et al. 2020). The inverse correlation between $\eta$ and $\Sigma_{*}$ is clear from observations and simulations. However, our empirical result supports that the radiative cooling below $10^{4} \mathrm{~K}$ is important. This is because when ignoring gas cooling below $10^{4} \mathrm{~K}$, there is an excess of warm gas compared with the models including a multiphase cold/warm gas ( $\mathrm{Li}$ et al. 2017). Thus, in the warm gas excess scenario, there is a layer of gas with higher ISM pressure which prevents the gas to be expelled from the supernova explosions.

\section{LOCAL TO GLOBAL MASS-LOADING FACTORS}

The mass-loading factor derived here is representative of local scales. However, other observational and theoretical studies report global mass-loading factors (Muratov et al. 2015; Rodríguez-Puebla et al. 2016; Hayward \& Hopkins 2017; Schroetter et al. 2019; McQuinn et al. 2019). We estimate global mass-loading factors, $\eta_{\mathrm{G}}$, from the empirical $\eta-\Sigma_{*}$ relation reported here (Eq. 6), integrating over observed stellar mass density profiles.

To convert $\eta$ to $\eta_{\mathrm{G}}$, we assume that we can estimate the total outflow due to stellar feedback, $\dot{M}_{\text {out }}$, by adding up $\dot{\Sigma}_{\text {out }}$ over each individual segment where stellar feedback acts. Since we are interested in galaxy discs, we assume a radial characterization for the properties of interest, i.e., $\eta$, $\dot{\Sigma}_{\text {out }}, \Sigma_{\mathrm{SFR}}$, and $\Sigma_{\mathrm{SFR}}$ depend on $R$, the radial distance to the centre of the disc:

$\eta_{\mathrm{G}}=\frac{\dot{M}_{\mathrm{out}}}{\mathrm{SFR}}=\frac{\int_{0}^{\infty} \dot{\Sigma}_{\mathrm{out}}(R) R \mathrm{~d} R}{\int_{0}^{\infty} \Sigma_{\mathrm{SFR}}(R) R \mathrm{~d} R}=\frac{\int_{0}^{\infty} \eta(R) \Sigma_{\mathrm{SFR}}(R) R \mathrm{~d} R}{\int_{0}^{\infty} \Sigma_{\mathrm{SFR}}(R) R \mathrm{~d} R}$.

The conversion between $\eta$ to $\eta_{\mathrm{G}}$ is a $\Sigma_{\mathrm{SFR}}$ weighted average of $\eta$. Stellar mass density profiles, $\Sigma_{*}(R)$, are better constrained than $\Sigma_{\mathrm{SFR}}(R)$ profiles, so we decide to use the empirical relation between $\Sigma_{\mathrm{SFR}} \propto \Sigma_{*}(R)^{n}$. For simplicity, we assume $n=1$, which is consistent with the latest results of this relation for galaxy discs (Cano-Díaz et al. 2019), although we found that different $n$ values close to 1 do not change the results significantly. Using our empirical relation (Eq. 6) we rewrite Eq. 7:

$\eta_{\mathrm{G}}=\frac{\int_{0}^{\infty} \eta(R) \Sigma_{*}(R) R \mathrm{~d} R}{\int_{0}^{\infty} \Sigma_{*}(R) R \mathrm{~d} R}=\frac{10^{3.2} \int_{0}^{\infty} \Sigma_{*}(R)^{0.68} R \mathrm{~d} R}{\int_{0}^{\infty} \Sigma_{*}(R) R \mathrm{~d} R}$.

The stellar mass surface density profile, $\Sigma_{*}(R)$, is therefore all we need to compute the global mass-loading factor. We use the deepest stellar mass surface density profiles from the Spitzer Survey of Stellar Structure in Galaxies (S4G) (Díaz-García et al. 2016) to compute the global mass-loading factor as a function of stellar mass. S4G stellar mass surface density profiles are divided into 5 mass bins: $\left[10^{8.5}-10^{9}\right]$, $\left[10^{9}-10^{9.5}\right],\left[10^{9.5}-10^{10}\right],\left[10^{10}-10^{10.5}\right]$, and $\left[10^{10.5}-10^{11}\right] \mathrm{M}_{\odot}$. We used these 5 mass bins to compute the $\eta_{\mathrm{G}}$ shown in Fig. 5.

\subsection{Variations of $\eta_{\mathrm{G}}$}

We present our empirically derived global mass-loading factors as a function of stellar mass in Fig. 5 as black lines. The discontinuity is due to the division in different parametrizations of the stellar mass surface density profiles in 5 mass 


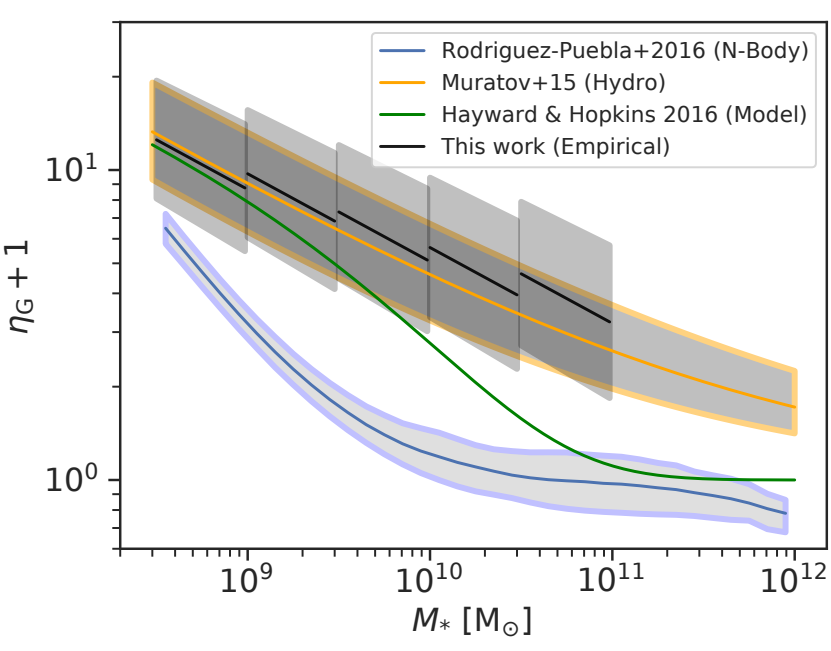

Figure 5. Global mass-loading factor, $\eta_{\mathrm{G}}$, versus the stellar mass of the galaxy, $\boldsymbol{M}_{*}$. The black lines show the empirical quantities which have been derived combining the distribution of $\eta$ as a function of stellar mass surface density (Fig. 4) with the stellar mass surface density profiles from S4G results (Díaz-García et al. 2016), where the shaded region is the corresponding uncertainty propagated from the $1-\sigma$ uncertainty found in the $\eta-\Sigma_{*}$ relation (Eq. 6). The discontinuity is due to the division in different parametrizations of the stellar mass surface density profiles in 5 mass bins reported by Díaz-García et al. (2016). The orange line shows the $\eta_{\mathrm{G}}$ reported for cosmological zoom-in galaxy hydrodynamical simulations (Muratov et al. 2015), the green line the shows an analytical feedback model (Hayward \& Hopkins 2017), and the blue line shows the $\eta_{\mathrm{G}}$ reported using the N-body BolshoiPlanck simulation (Rodríguez-Puebla et al. 2016).

bins reported by Díaz-García et al. (2016) . The most important feature we find in Fig. 5 is that the smaller the stellar mass of the galaxy, the larger the mass-loading factor, as required to reconcile the ratio of halo to stellar mass in lowmass galaxies (Behroozi et al. 2010; Rodríguez-Puebla et al. 2016). We compare our empirical estimates with predictions based on N-body (Rodríguez-Puebla et al. 2016) and hydrodynamical simulations (Muratov et al. 2015), as well as with an analytical model (Hayward \& Hopkins 2017).

Some simulations, as well as the analytic model we used to compare with, define the mass-loading factor using the outflowing mass that escapes the galaxy forever (RodríguezPuebla et al. 2016; Hayward \& Hopkins 2017). Therefore, they do not consider the outflowing gas which returns to the galaxy at a later time which, depending on the outflow velocity, will result in smaller mass-loading factors. We define the mass-loading factor as the total mass ejected independently of velocity, and the mass can come back at a later time (outside the $550 \mathrm{Myr}$ time range). There is a remarkable agreement between our empirical result and that from hydrodynamical simulations that define the mass-loading factor as independent of velocity (Muratov et al. 2015). In fact, for lower masses $\left(M_{*}<10^{9.5} \mathrm{M}_{\odot}\right)$, where the stellar feedback is thought to be more important regulating galaxy stellar mass growth, and the escape velocity lower, the agreement with the analytic model is also remarkably good.

\section{DISCUSSION}

\subsection{Comparison with other studies}

The results reported here are slightly different when we compare them with recent observational studies reporting local (Kruijssen et al. 2019; Roberts-Borsani et al. 2020) and global (Schroetter et al. 2019; McQuinn et al. 2019) massloading factors.

The reported local mass-loading factors using the spatial de-correlation between star formation and molecular gas (Kruijssen et al. 2019) differ by less than $3 \sigma$ from our reported values, and it might be due to the smaller time scale of $\sim 1.5 \mathrm{Myr}$ for which they report efficient gas dispersal, while our reported time scale is $\sim 550 \mathrm{Myr}$. The previously reported local mass-loading factors using the Na D absorption (Roberts-Borsani et al. 2020) are consistent with ours within $1 \sigma$.

The use of $\mathrm{Mg}$ II absorption of the circum-galactic medium to derive global mass-loading factors gives no clear dependence on the total mass of the galaxy (Schroetter et al. 2019). However, the Mg II absorption method gives $\eta_{\mathrm{G}}$ with very high uncertainties, mainly due to the uncertainty when deriving the HI column density from the $\mathrm{Mg}$ II equivalent width (Schroetter et al. 2015). Due to these large uncertainties, their results are apparently consistent with our results within $1 \sigma$ for most of their reported $\eta_{\mathrm{G}}$ 's.

The mass-loading factor estimates using deep $\mathrm{H} \alpha$ imaging give smaller mass-loading factors compared to those reported here and give no correlation with the stellar mass of the galaxy (McQuinn et al. 2019). Nevertheless, the method using $\mathrm{H} \alpha$ imaging derives the amount of outflowing gas from the $\mathrm{H} \alpha$ surface brightness background, so it neglects $\mathrm{H} \alpha$ emission stronger than this background emission. The estimated outflowing mass could be inferior to the real one since we already know that $\mathrm{H} \alpha$ emission has a component due to expansive bubbles (Relaño \& Beckman 2005; Camps-Fariña et al. 2015).

\subsection{Discussion on envelope's shapes}

Eq. 5 depends on $\dot{\Sigma}_{\text {flow max }}, \eta, \Sigma_{\text {SFR recent }}$, and $\Sigma_{\text {SFR past }}$ values. The case shown in Fig. 3, where there is no high recent star formation rate for those regions where the past star formation rate was the highest, is a common case, but not the only one. For instance, there are cases where $\Sigma_{\text {SFR recent }}$ values are approximately constant through the regions on the envelope or even increase as $\Sigma_{\text {SFR past }}$ increase (e.g. NGC 988, NGC 7421, IC 217, IC 4452, PGC 28308 in Fig. A1).

Essentially, there are two terms depending on $\Sigma_{\text {SFR past }}$ in Eq. 5, one with a direct proportionality and the other with an inverse one. The latter dominates for larger $\eta$ values meaning that the larger the mass-loading factor, the larger is the effect in reducing the amount of gas to form stars, as expected. However, for small enough $\eta$ and $\Sigma_{\text {SFR past }}$ values, the directly dependent term can dominate producing a direct relation between $\Sigma_{\text {SFR recent }}$ and $\Sigma_{\text {SFR past }}$, as we see in some galaxies in Fig. A1 (e.g. IC 4452). The extrapolation of Eq. 5 to large enough values of $\Sigma_{\text {SFR past }}$ would give always a decrease in $\Sigma_{\text {SFR recent }}$, as long as $\eta>0$, and that is the reason to observe some inverted U-shape envelopes (e.g. NGC 1084, PGC 3140). 


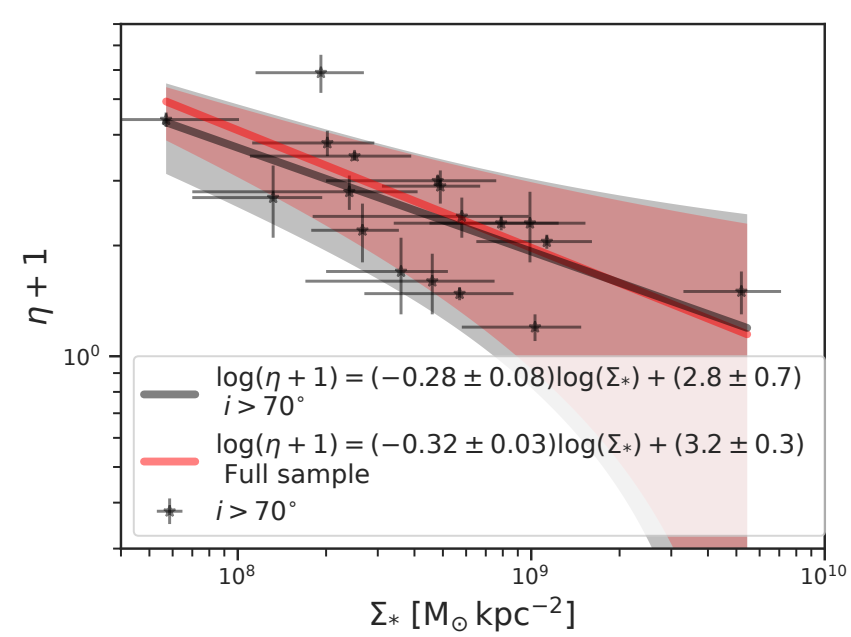

Figure 6. Local mass-loading factor, $\eta$, versus the average stellar mass surface density of the regions on the envelope for each galaxy, $\Sigma_{*}$, as black stars, for high-inclination galaxies. We plot, as a black line, the linear fit to the empirically derived quantities. We plot as a red line, the fit for the full sample (Eq. 6), and the $1-\sigma$ uncertainty range of the fits as shaded regions.

Finally, for large enough $\dot{\Sigma}_{\text {flow max }}$ values, the directly $\Sigma_{\text {SFR past }}$ dependent term is almost negligible for small values of $\Sigma_{\text {SFR past }}$, and then the recent star formation depends on $\dot{\Sigma}_{\text {flow max }}$ for the low $\Sigma_{\text {SFR past }}$, while decreases proportionally with $\Sigma_{\text {SFR past }}$ depending on the mass-loading factor (e.g UGC 5378, UGC 11001).

Therefore, the combination of $\dot{\Sigma}_{\text {flow max }}$ and $\eta$ variations, as well as the range covered by the past and recent star formation rate surface densities, is what gives the different envelope's shapes. The uncertainties obtained when fitting Eq. 5 show reliable estimates, except for one case, the PGC 30591 galaxy, which is the one having the highest inclination of our sample.

\subsection{High-inclination galaxies}

Although we have removed edge-on $\left(i=90^{\circ}\right)$ galaxies from our sample, high-inclination $\left(i>70^{\circ}\right)$ galaxies might not be good candidates to apply the method used in this study, as in the case of the PGC 30591 galaxy. In the case of edge-on and very high-inclination galaxies, two main effects can affect the pertinence of applying the method. Firstly, one can have in the line of sight a large combination of different regions of the galaxy. Secondly, the higher the inclination, the smaller is the number of resolved regions, while the method relies on a large enough number of resolved regions.

Nevertheless, except for PGC 30591, we found reliable $\dot{\Sigma}_{\text {flow max }}$ and $\eta$ estimates, even for high-inclination galaxies. Including or excluding high-inclination galaxies does not make any changes to the reported $\eta$ - $\Sigma_{*}$ relation presented here. We show in Fig. 6 the $\eta$ - $\Sigma_{*}$ observed and fitted relations for high-inclination galaxies, as star symbols and black line, respectively. When we compare the high-inclination galaxies results with the fit obtained using the full sample (Eq. 6), shown as a red line, both samples are compatible within $1 \sigma$.

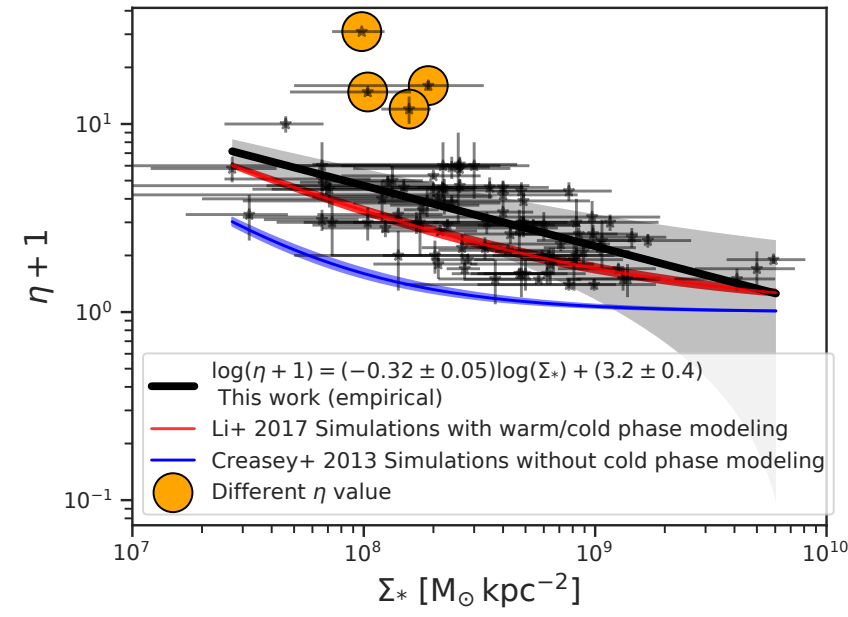

Figure 7. Local mass-loading factor, $\eta$, versus the average stellar mass surface density of the regions on the envelope for each galaxy, $\Sigma_{*}$, as black stars. Same plot as in Fig. 4, but this version of the plot has been done without removing any region based on its $\Sigma_{\text {SFR recent }}$ value. The 4 galaxies where we find a significant difference in the derived $\eta$ value are marked as orange circles. We plot the linear fit to the empirically derived quantities and the $1-\sigma$ uncertainty range of the fit as shaded regions. The blue line shows the $\eta$ reported for supernova explosions hydrodynamical simulations (Creasey et al. 2013) with cut-off cooling modeling, and as blue line the $\boldsymbol{\eta}$ reported for supernova explosions hydrodynamical simulations ( $\mathrm{Li}$ et al. 2017) with gas cold-phase modeling included.

\subsection{Effects of very high recent SFR regions removal}

We think is important to remove regions having very high values of $\Sigma_{\text {SFR recent }}$ compared to the rest of the galaxy, since these regions probably have a very high $\dot{\Sigma}_{\text {net flow value com- }}$ pared to the rest of the regions identified to be on the envelope. The effect of considering these high $\Sigma_{\text {SFR recent }}$ regions affects our assumption about the approximate equal value of $\dot{\Sigma}_{\text {net flow }}$ for the regions on the envelope.

However, in order to explore the effect of this removal in our results, we have performed the same analysis without removing any region based on its $\Sigma_{\text {SFR recent value. We plot }}$ the derived $\eta$ versus the local $\Sigma_{*}$ of the regions on the envelope for the whole sample of galaxies in Fig. 7. We also plot the resulted $\eta-\Sigma_{*}$ fit to the observed data. We find a very similar correlation between $\eta$ and the local $\Sigma_{*}$ :

$\log (\eta+1)=(-0.32 \pm 0.05) \log \left(\Sigma_{*}\right)+(3.2 \pm 0.4)$.

There are only 4 galaxies where the $\eta$ value significantly changes: IC 1158, NGC 3389, ESO 298-G28, and MCG 0157-021. We marked these galaxies as orange circles in Fig. 7. However, the changes are not significant enough to change the resulted $\eta-\Sigma_{*}$ relation, but just a slightly increase in the obtained uncertainties. Therefore, although the removal could be important for some specific individual galaxies, when compared with the full sample of galaxies we still find a consistent $\eta-\Sigma_{*}$ relation. 


\section{CONCLUSIONS}

We have used MUSE observations of a sample of 102 galaxy discs. We extracted the spectra of $500 \mathrm{pc}$ wide regions and apply them stellar population synthesis using SINOPSIS code. We obtained the star formation histories of those regions and we analysed the recent and past star formation rate densities. We compared the $\Sigma_{\text {SFR recent }}$ with the $\Sigma_{\text {SFR past }}$ and found that, for each galaxy, there is an envelope of regions formed by those regions having the maximum $\Sigma_{\text {SFR recent }}$, per bin of $\Sigma_{\text {SFR past }}$. We fitted the resolved star formation self-regulator model (Eq. 5) to those regions on the envelope and quantify the mass-loading factor, $\eta$.

We find correlations locally between $\eta$ and the stellar mass surface density, $\Sigma_{*}$, and globally between the averaged value of $\eta$ for a galaxy, $\eta_{\mathrm{G}}$, and the stellar mass of the galaxy, $M_{*}$, which are strong indications of how stellar feedback locally regulates the mass growth of galaxies, especially those of lower masses. The comparison between our empirical local $\eta-\Sigma_{*}$ relation with that from hydrodynamical simulations of supernova explosions ( $\mathrm{Li}$ et al. 2017) is remarkably in agreement. In the case of our empirical global $\eta_{\mathrm{G}}-M_{*}$ relation, the comparison with hydrodynamical cosmological zoom-in galaxy simulations (Muratov et al. 2015) is also in excellent agreement.

We note that the value of $\eta$ depends on the time scale over which the feedback is analysed, and can be defined either including or excluding posterior gas return, so comparison with other observations and with theory must be done with care. These empirical relations offer excellent tools to confront with stellar feedback models which are crucial for understanding galaxy formation and evolution.

\section{ACKNOWLEDGEMENTS}

The authors thank the anonymous referee whose comments have led to significant improvements in the paper. The authors also thank Aldo Rodríguez-Puebla for sharing their stellar halo accretion rate coevolution models from Rodríguez-Puebla et al. (2016). JZC and IA's work is funded by a CONACYT grant through project FDC-2018-1848. DRG acknowledges financial support through CONACYT project A1-S-22784. GB acknowledges financial support through PAPIIT project IG100319 from DGAPA-UNAM. This research has made use of the services of the ESO Science Archive Facility, Astropy, ${ }^{4}$ a community-developed core Python package for Astronomy (Astropy Collaboration et al. 2013, 2018), APLpy, an open-source plotting package for Python (Robitaille \& Bressert 2012), Astroquery, a package that contains a collection of tools to access online Astronomical data (Ginsburg et al. 2019), and pyregion (https:// github.com/astropy/pyregion), a python module to parse ds9 region files. We acknowledge the usage of the HyperLeda database (http://leda.univ-lyon1.fr). Based on observations collected at the European Southern Observatory under ESO programmes 095.D-0172(A), 196.B-0578(B), 096.D0263(A), 097.D-0408(A), 095.B-0532(A), 096.D-0296(A), 60.A-9319(A), 1100.B-0651(B), 0102.B-0794(A), 098.B0551(A), 0101.D-0748(A), 099.B-0397(A), 1100.B-0651(A),

4 http://www.astropy.org
0100.D-0341(A), 096.B-0309(A), 296.B-5054(A), 095.D0091(B), 0101.D-0748(B), 095.D-0091(A), 097.B-0640(A), 096.B-0054(A), 1100.B-0651(C), 098.D-0115(A), 097.B0165(A), 098.C-0484(A), 094.C-0623(A), 60.A-9339(A), 099.B-0242(A), 0102.A-0135(A), 097.B-0041(A), 095.B0934(A), 099.D-0022(A), and 60.A-9329(A).

\section{REFERENCES}

Arp H., 1966, ApJS, 14, 1

Ascasibar Y., Gavilán M., Pinto N., Casado J., Rosales-Ortega F., Díaz A. I., 2015, MNRAS, 448, 2126

Astropy Collaboration et al., 2013, A\&A, 558, A33

Astropy Collaboration et al., 2018, AJ, 156, 123

Bacon R., et al., 2010, in Proc. SPIE. p. 773508, doi:10.1117/12.856027

Barrera-Ballesteros J. K., et al., 2018, ApJ, 852, 74

Barrera-Ballesteros J. K., et al., 2020, MNRAS, 492, 2651

Behroozi P. S., Conroy C., Wechsler R. H., 2010, ApJ, 717, 379

Bigiel F., Leroy A., Walter F., Brinks E., de Blok W. J. G., Madore B., Thornley M. D., 2008, AJ, 136, 2846

Bouché N., et al., 2010, ApJ, 718, 1001

Calzetti D., Armus L., Bohlin R. C., Kinney A. L., Koornneef J., Storchi-Bergmann T., 2000, ApJ, 533, 682

Camps-Fariña A., Zaragoza-Cardiel J., Beckman J. E., Font J., García-Lorenzo B., Erroz-Ferrer S., Amram P., 2015, MNRAS, 447, 3840

Cano-Díaz M., Ávila-Reese V., Sánchez S. F., Hernández-Toledo H. M., Rodríguez-Puebla A., Boquien M., Ibarra-Medel H., 2019, MNRAS, 488, 3929

Chabrier G., 2003, PASP, 115, 763

Cid Fernandes R., Mateus A., Sodré L., Stasińska G., Gomes J. M., 2005, MNRAS, 358, 363

Creasey P., Theuns T., Bower R. G., 2013, MNRAS, 429, 1922

Dekel A., Mandelker N., 2014, MNRAS, 444, 2071

Díaz-García S., Salo H., Laurikainen E., 2016, A\&A, 596, A84

Erroz-Ferrer S., et al., 2019, MNRAS, 484, 5009

Ferland G. J., 1993, Hazy, A Brief Introduction to Cloudy 84

Ferland G. J., Korista K. T., Verner D. A., Ferguson J. W., Kingdon J. B., Verner E. M., 1998, PASP, 110, 761

Ferland G. J., Kisielius R., Keenan F. P., van Hoof P. A. M., Jonauskas V., Lykins M. L., Porter R. L., Williams R. J. R., 2013, ApJ, 767, 123

Forbes J. C., Krumholz M. R., Burkert A., Dekel A., 2014, MNRAS, 443, 168

Fraternali F., 2017, Gas Accretion via Condensation and Fountains. p. 323, doi:10.1007/978-3-319-52512-9_14

Fritz J., et al., 2007, A\&A, 470, 137

Fritz J., et al., 2011, A\&A, 526, A45

Fritz J., et al., 2017, ApJ, 848, 132

Ginsburg A., et al., 2019, AJ, 157, 98

Hayward C. C., Hopkins P. F., 2017, MNRAS, 465, 1682

Hickson P., 1982, ApJ, 255, 382

Hopkins P. F., Kereš D., Oñorbe J., Faucher-Giguère C.-A., Quataert E., Murray N., Bullock J. S., 2014, MNRAS, 445, 581

Hopkins P. F., et al., 2018, MNRAS, 480, 800

Kennicutt Robert C. J., et al., 2007, ApJ, 671, 333

Kewley L. J., Groves B., Kauffmann G., Heckman T., 2006, MNRAS, 372, 961

Kormendy J., Kennicutt Robert C. J., 2004, ARA\&A, 42, 603

Kreckel K., et al., 2019, ApJ, 887, 80

Kruijssen J. M. D., et al., 2019, Nature, 569, 519

Lada C. J., Lada E. A., 2003, ARA\&A, 41, 57

Leroy A. K., et al., 2019, ApJS, 244, 24

Li M., Bryan G. L., Ostriker J. P., 2017, ApJ, 841, 101 
Lilly S. J., Carollo C. M., Pipino A., Renzini A., Peng Y., 2013, ApJ, 772, 119

López-Cobá C., et al., 2020, AJ, 159, 167

Madau P., Dickinson M., 2014, ARA\&A, 52, 415

Makarov D., Prugniel P., Terekhova N., Courtois H., Vauglin I., 2014, A\&A, 570, A13

Martín-Navarro I., Brodie J. P., Romanowsky A. J., Ruiz-Lara T., van de Ven G., 2018, Nature, 553, 307

McQuinn K. B. W., van Zee L., Skillman E. D., 2019, ApJ, 886, 74

Muratov A. L., Kereš D., Faucher-Giguère C.-A., Hopkins P. F., Quataert E., Murray N., 2015, MNRAS, 454, 2691

Osterbrock D. E., 1989, Astrophysics of gaseous nebulae and active galactic nuclei

Paturel G., Petit C., Prugniel P., Theureau G., Rousseau J., Brouty M., Dubois P., Cambrésy L., 2003, A\&A, 412, 45

Poggianti B. M., et al., 2017, ApJ, 844, 48

Relaño M., Beckman J. E., 2005, A\&A, 430, 911

Roberts-Borsani G. W., Saintonge A., Masters K. L., Stark D. V., 2020, MNRAS, 493, 3081

Robitaille T., Bressert E., 2012, APLpy: Astronomical Plotting Library in Python (ascl:1208.017)

Rodríguez-Puebla A., Primack J. R., Behroozi P., Faber S. M., 2016, MNRAS, 455, 2592

Sánchez-Menguiano L., et al., 2018, A\&A, 609, A119

Sánchez S. F., et al., 2016, Rev. Mex. Astron. Astrofis., 52, 21

Schaye J., et al., 2010, MNRAS, 402, 1536

Schroetter I., Bouché N., Péroux C., Murphy M. T., Contini T., Finley H., 2015, ApJ, 804, 83

Schroetter I., et al., 2019, MNRAS, 490, 4368

Silk J., Mamon G. A., 2012, Research in Astronomy and Astrophysics, 12, 917

Somerville R. S., Davé R., 2015, ARA\&A, 53, 51

Vogelsberger M., Genel S., Sijacki D., Torrey P., Springel V., Hernquist L., 2013, MNRAS, 436, 3031

Vorontsov-Velyaminov B. A., 1959, Atlas and Catalog of Interacting Galaxies (1959, p. 0

Werle A., Cid Fernandes R., Vale Asari N., Bruzual G., Charlot S., Gonzalez Delgado R., Herpich F. R., 2019, MNRAS, 483, 2382

Zaragoza-Cardiel J., et al., 2019, MNRAS, 487, L61

\section{DATA AVAILABILITY}

The data underlying this article are available in the ESO archive, at http://archive.eso.org/. The datasets were derived for each source through the query form at http://archive.eso.org/wdb/wdb/adp/phase3_ spectral/form?collection_name=MUSE.

\section{APPENDIX A: SFR RECENT-PAST \\ DIAGRAMS}



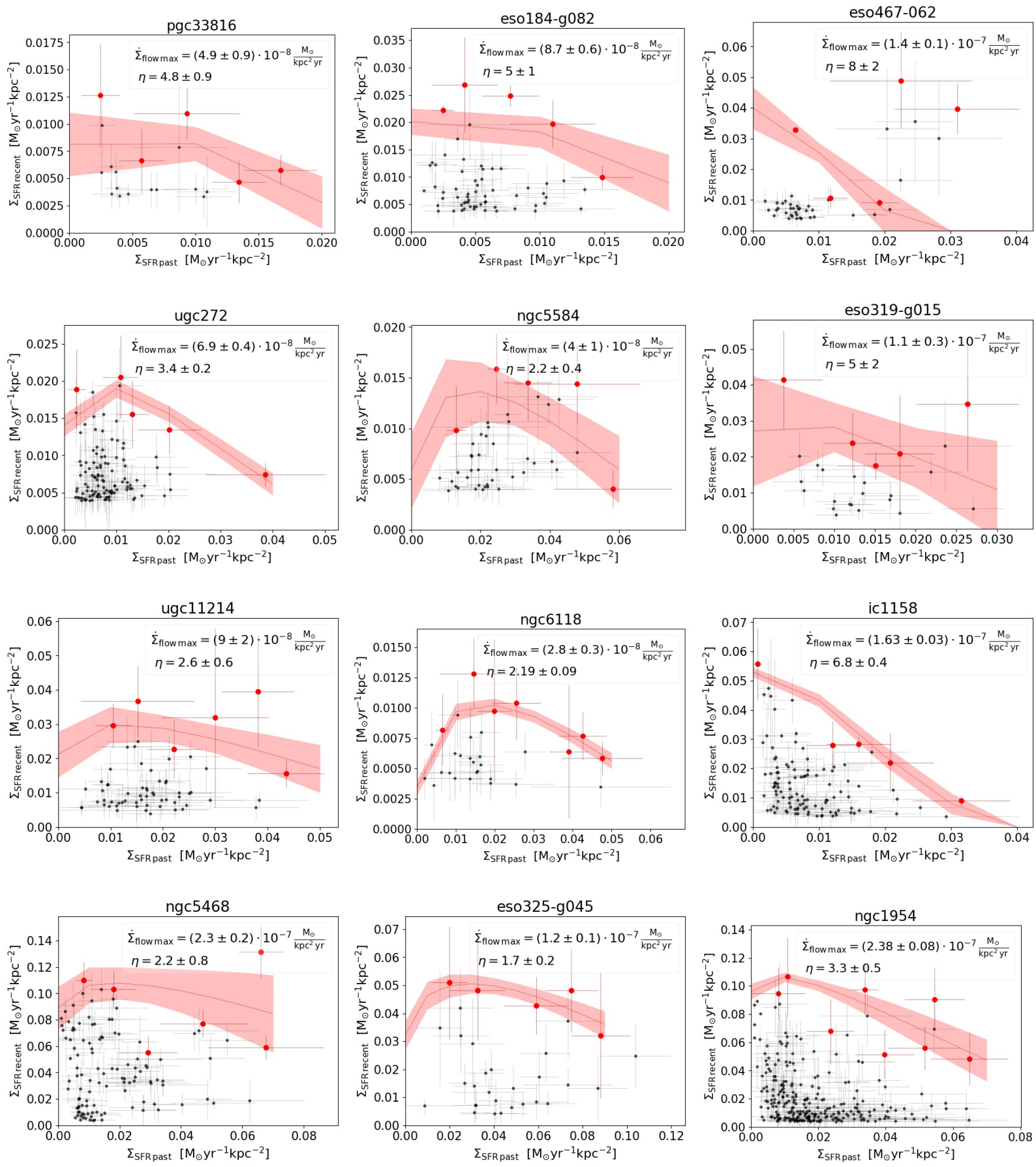

Figure A1. SFR recent-past diagrams. Recent star formation surface density, $\Sigma_{\text {SFR recent }}$, versus the past star formation rate surface density, $\Sigma_{\text {SFR past }}$, for each galaxy of the sample. The red dots are the regions identified as those on the envelope. We plot the fit of Eq. 5 to the regions on the envelope and the 1- $\sigma$ uncertainty range of the fit as shadow region. We show the parameters of the fit, at the top of each panel. 

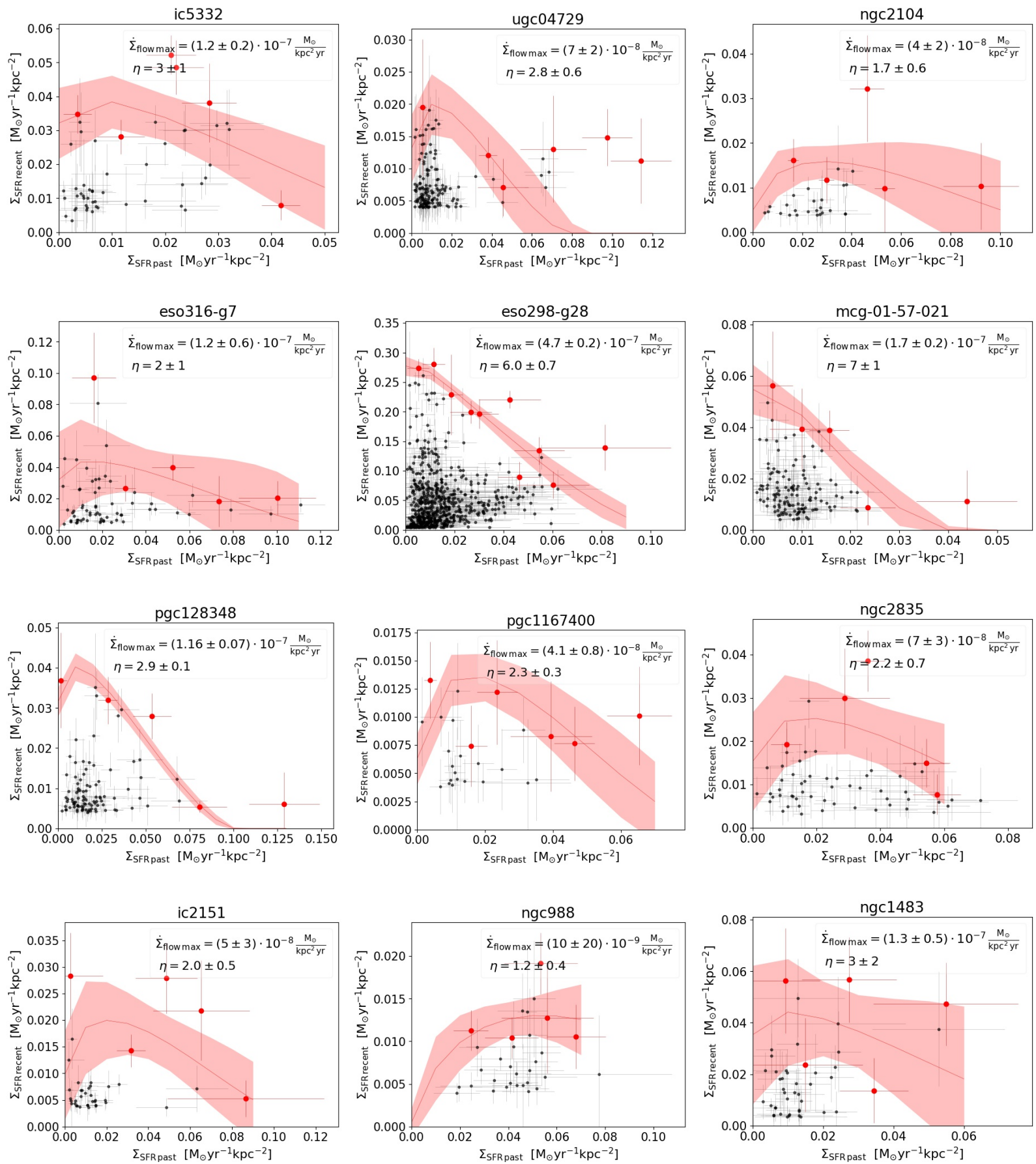

Figure A1 - continued 

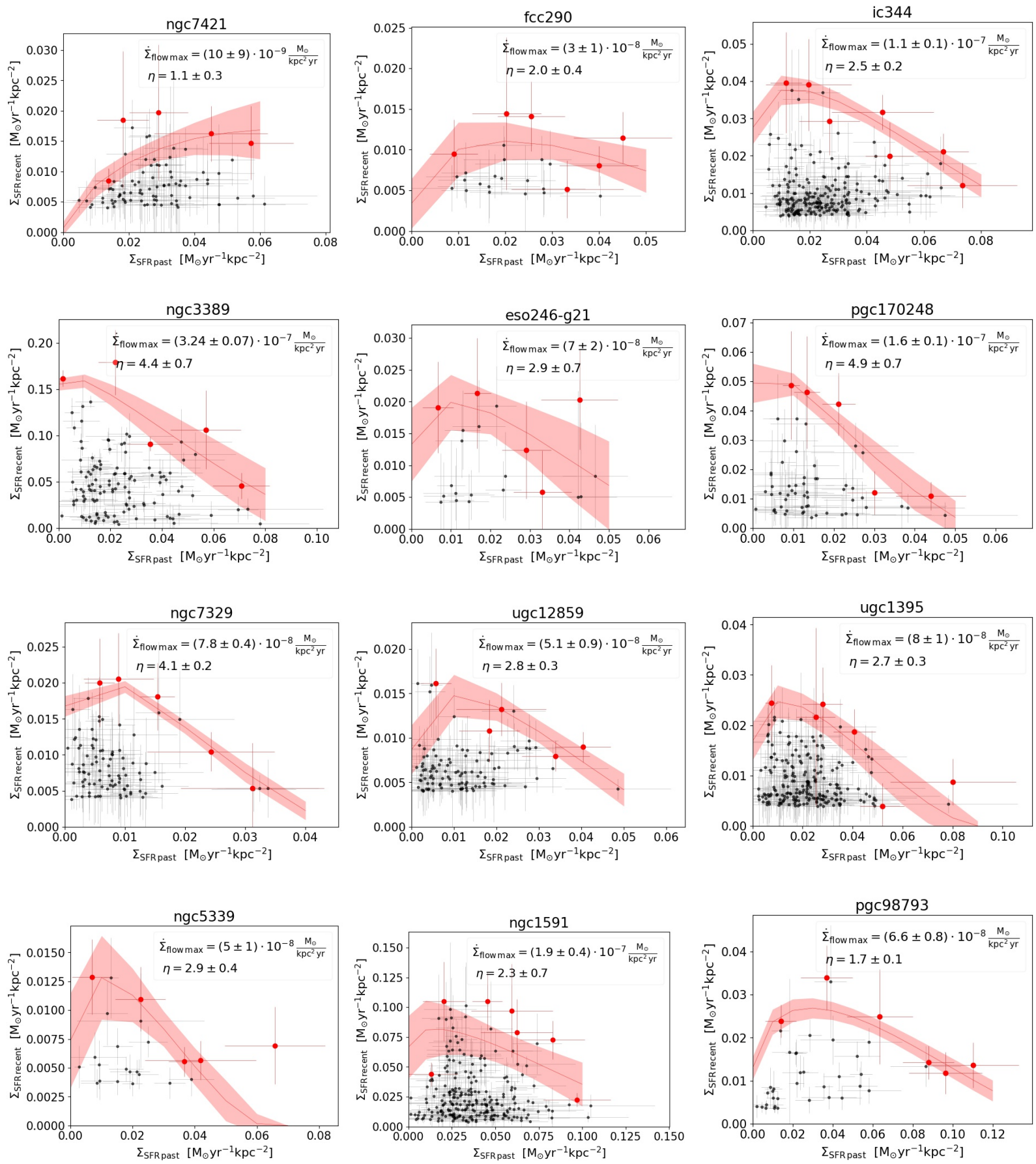

Figure A1 - continued 

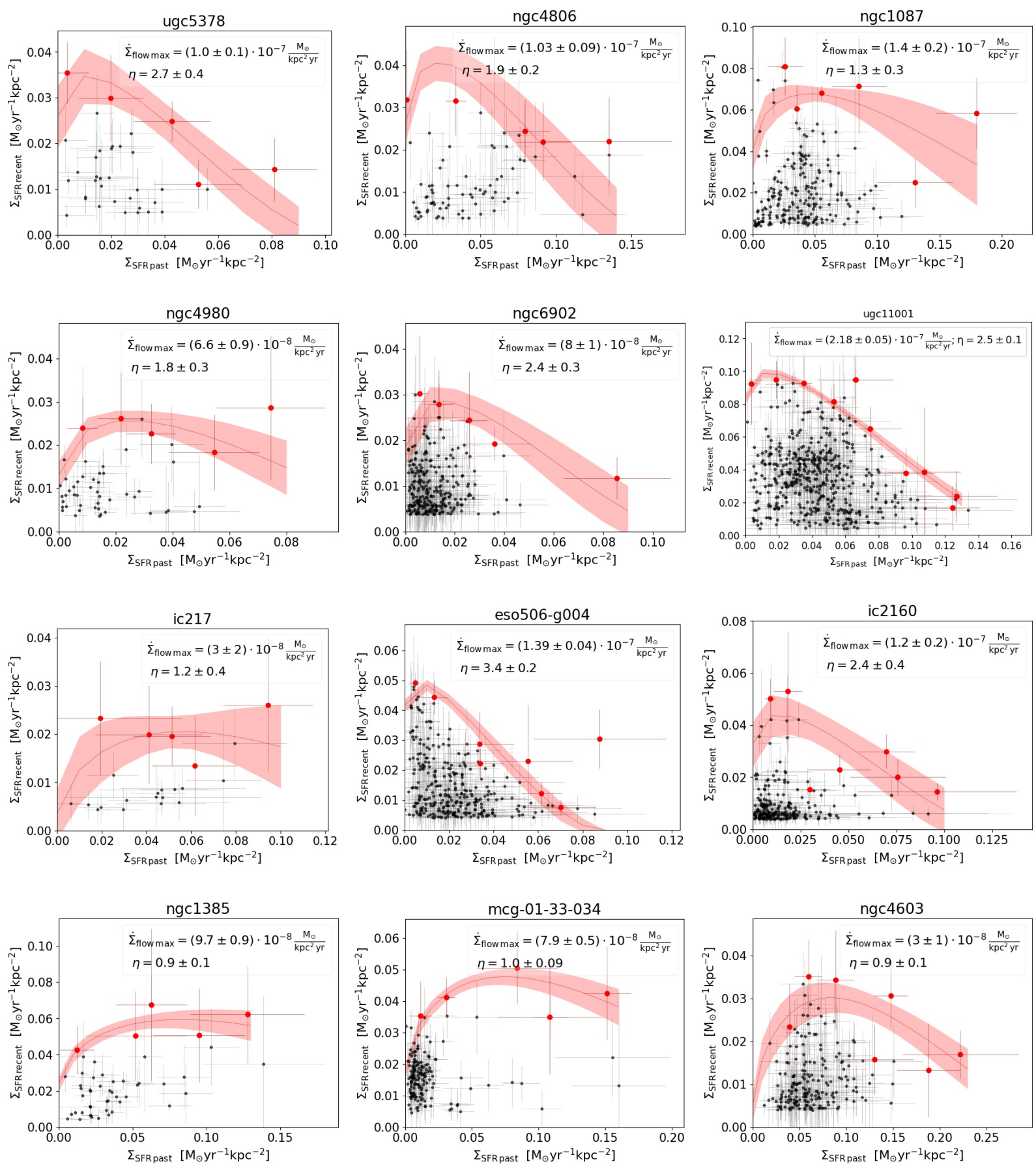

Figure A1 - continued 

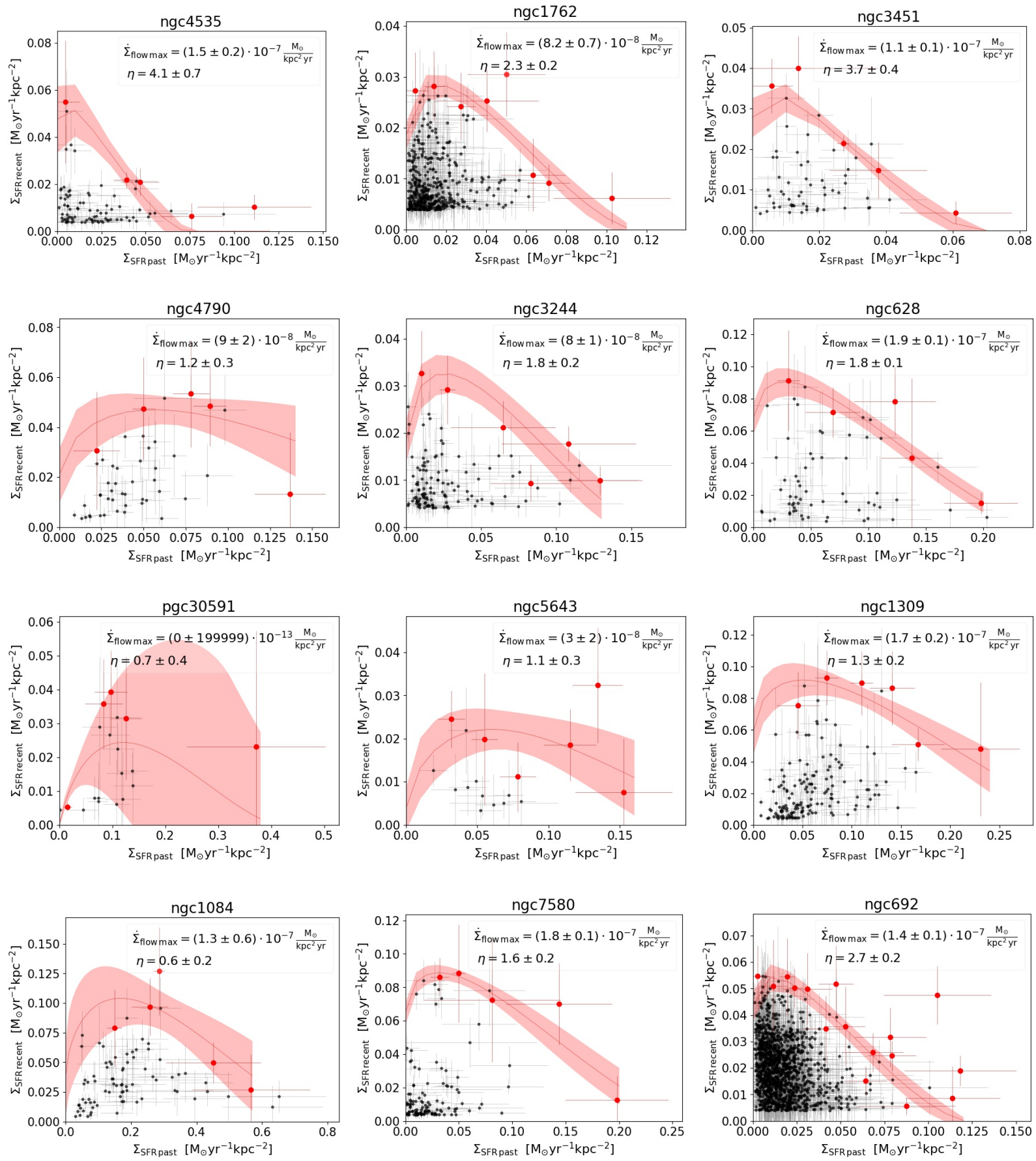

Figure A1 - continued 

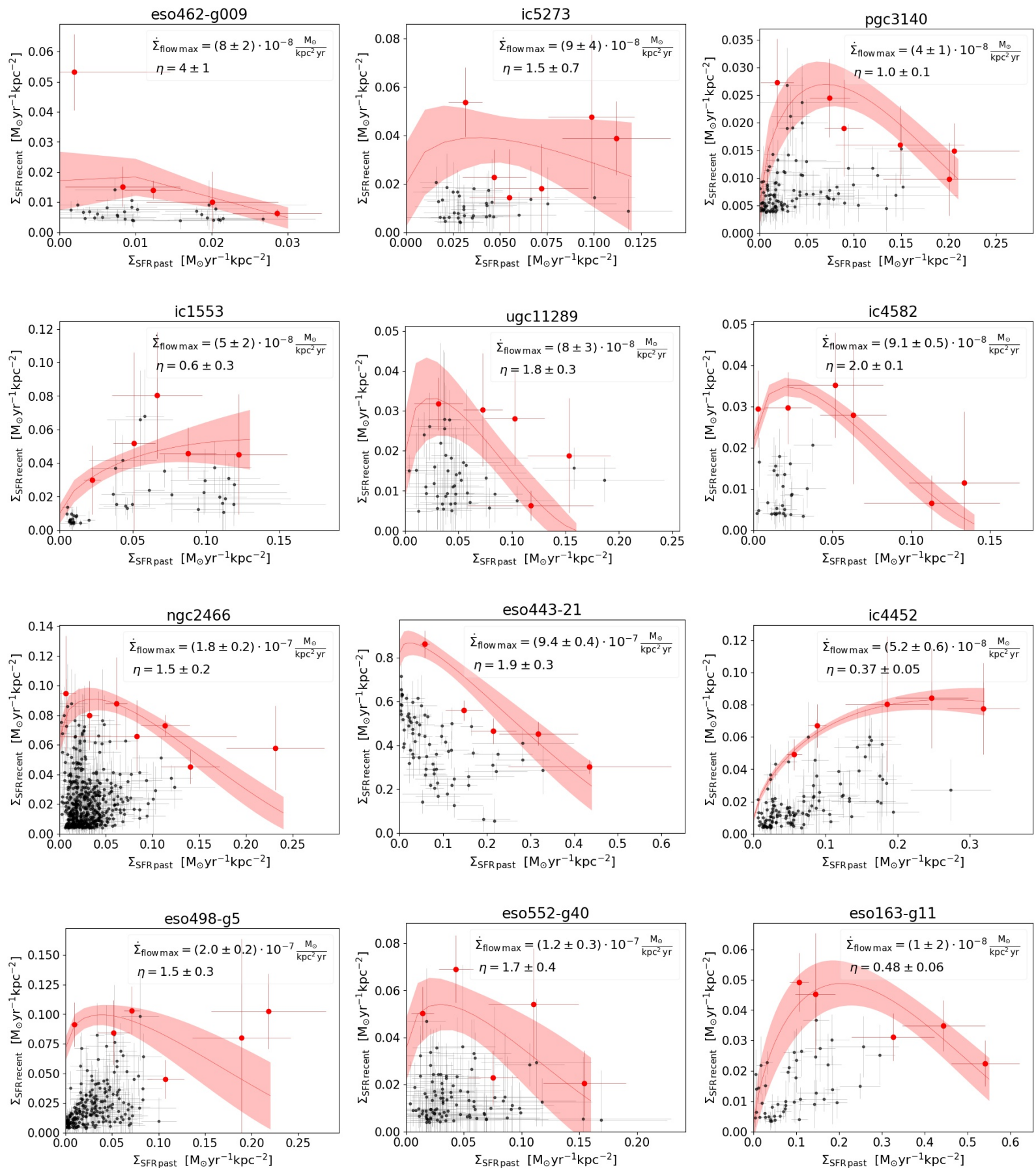

Figure A1 - continued 

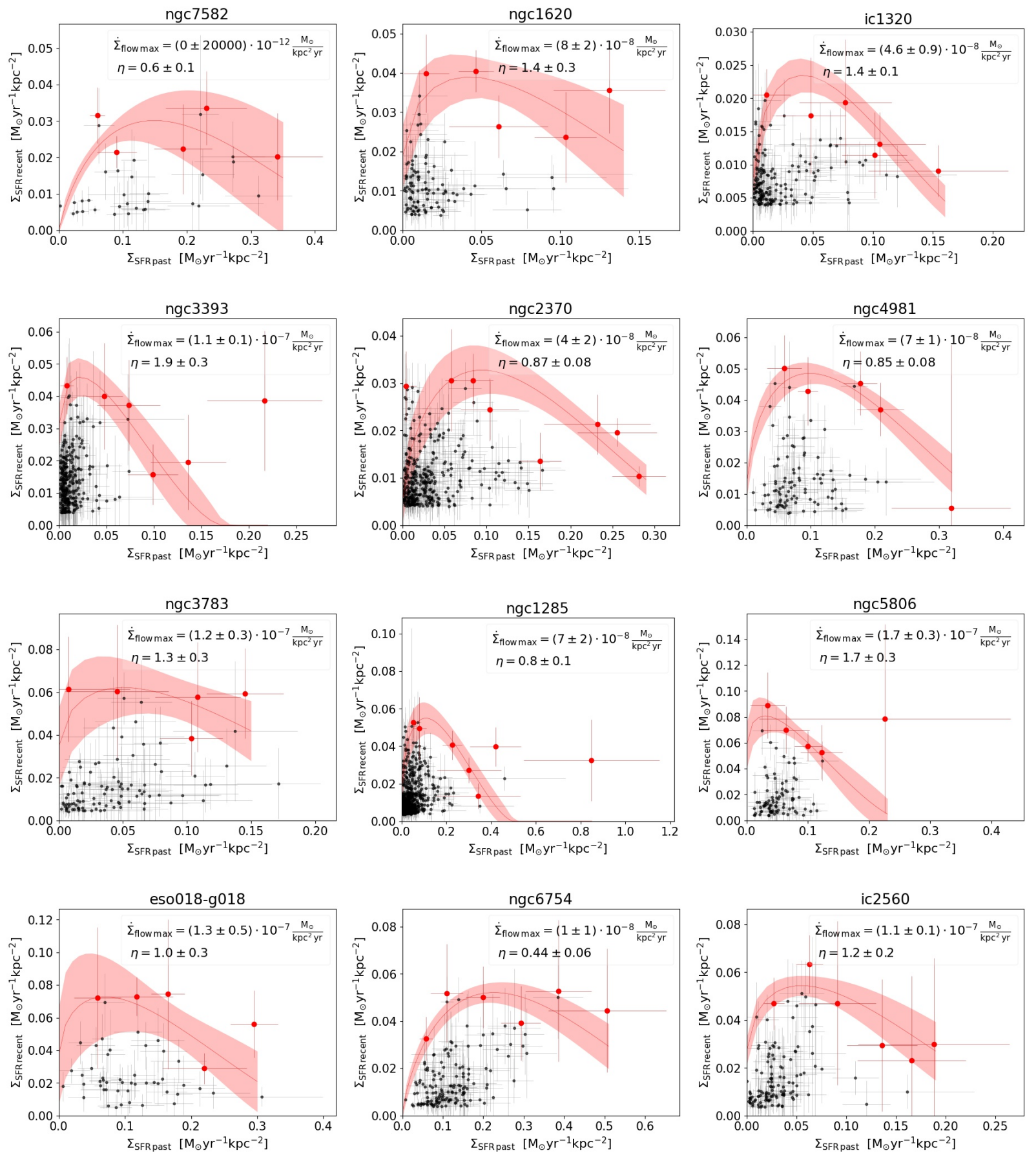

Figure A1 - continued 

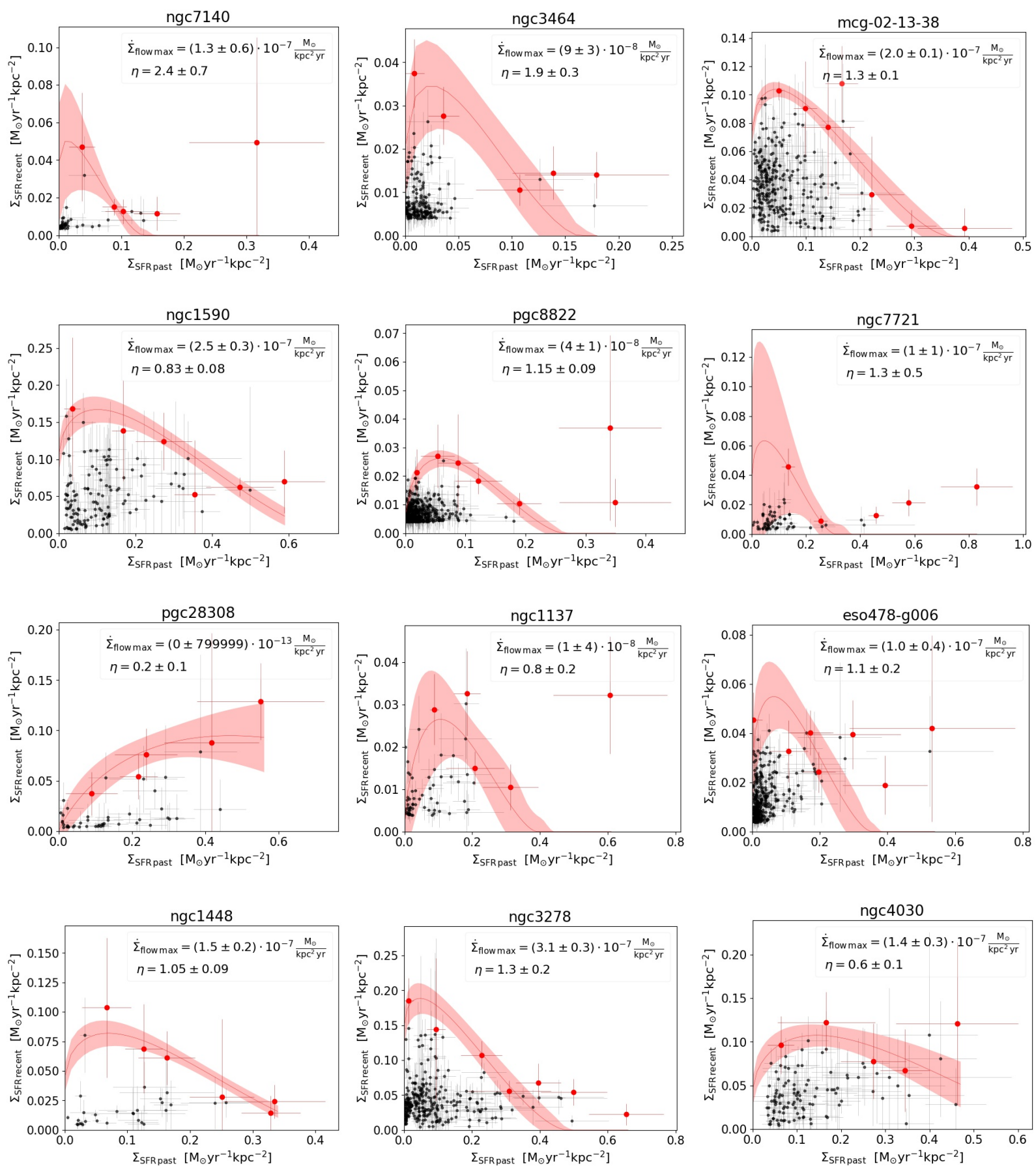

Figure A1 - continued 

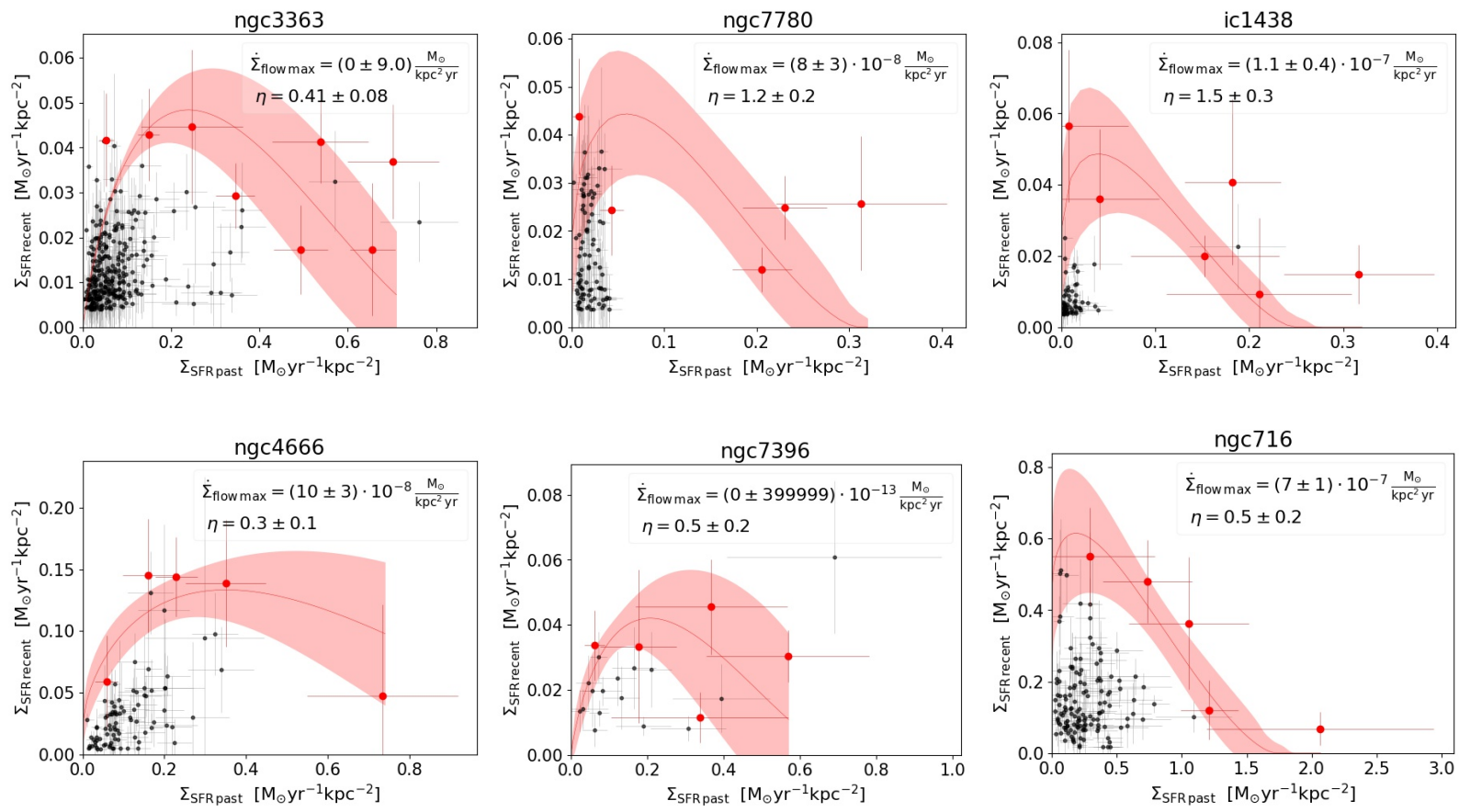

Figure A1 - continued 
22 J. Zaragoza-Cardiel et al.

This paper has been typeset from a $\mathrm{T}_{\mathrm{E}} \mathrm{X} / \mathrm{LAT}_{\mathrm{E}} \mathrm{X}$ file prepared by the author. 